\title{
A Coordinate Displacement Compensation-Based Quality Enhancement Method for Chroma Subsampling and Upsampling on VVC
}

\author{
Kuo-Liang Chung, Senior Member, IEEE, and Chen-Wei Kao
}

\begin{abstract}
Prior to encoding an input RGB full-color image $I^{R G B}$, at the server side, performing chroma subsampling on the converted chroma image is a necessary step. After receiving the decompressed subsampled chroma image and luma image at the client side, performing chroma upsampling is also a necessary step for reconstructing the RGB full-color image. In this paper, we consider seven commonly used chroma subsampling methods, denoted by $C_{s}$, and four widely used chroma upsampling methods, denoted by $C_{u}$. For each combination $c_{s}-c_{u}$ in $C_{s} \mathbf{x} C_{u}$, we first utilize the moment balance law to analyze the coordinate displacement (CD) bias problem occurring in $c_{s}$. Next, for the combination $c_{s}-c_{u}$, we analyze the $\mathrm{CD}$ bias problem occurring in the transition from the server side to the client side. Then, we explain why the CD bias problem degrades the quality of the reconstructed RGB full-color images in the current coding system. To remedy this CD bias problem, a CD compensationbased (CDC-based) quality enhancement method is proposed to improve the quality of the reconstructed images. To the best of our knowledge, this is the first work in this research direction. Based on the IMAX, Kodak, SCI (screen content images), and Video datasets, the comprehensive experimental results have demonstrated that on the newly released versatile video coding (VVC) platform VTM-12.0, the proposed CDC-based quality enhancement method in our augmented coding system can achieve substantial quality improvement for 17 combinations in $C_{s} \mathbf{x} C_{u}$.
\end{abstract}

Index Terms-Augmented coding system, Chroma subsampling, Chroma upsampling, Coordinate displacement bias, Moment balance law, Quality enhancement, Quality-bitrate tradeoff, RGB full-color image, Versatile video coding (VVC).

\section{INTRODUCTION}

$\mathbf{I}$ $\mathrm{N}$ this study, as depicted in Fig. 1, the input image is an RGB full-color image $I^{R G B}$. Prior to compression, according to BT.601-5 [3], $I^{R G B}$ is converted to a $\mathrm{YCbCr}$ image $I^{Y C b C r}$ using the following RGB-to-YCbCr color transformation:

$$
\left[\begin{array}{c}
Y_{i} \\
C b_{i} \\
C r_{i}
\end{array}\right]=\left[\begin{array}{ccc}
0.257 & 0.504 & 0.098 \\
-0.148 & -0.291 & 0.439 \\
0.439 & -0.368 & -0.071
\end{array}\right]\left[\begin{array}{c}
R_{i} \\
G_{i} \\
B_{i}
\end{array}\right]+\left[\begin{array}{c}
16 \\
128 \\
128
\end{array}\right]
$$

where for each $2 \times 2$ YCbCr block $B^{Y C b C r}$, each entry $\left(Y_{i}, C b_{i}, C r_{i}\right), 1 \leq i \leq 4$, denotes the YCbCr triple-value;

This work was supported by the contracts MOST-108-2221-E-011-077MY3 and 110-2221-E-011-088-MY3 of the Ministry of Science and Technology, Taiwan.

K. L. Chung (Corresponding author. E-mail: klchung01@gmail.com) and C. W. Kao are with the Department of Computer Science and Information Engineering, National Taiwan University of Science and Technology, Taipei 10672, Taiwan.
$\left(R_{i}, G_{i}, B_{i}\right)$ denotes the collocated RGB triple-value of the $2 \times 2$ RGB full-color block $B^{R G B}$.

As depicted at the server side of Fig. 1, a chroma subsampling method is performed on each $2 \times 2 \mathrm{CbCr}$ block $B^{C b C r}$ to obtain the subsampled $(\mathrm{Cb}, \mathrm{Cr})$-pair of $B^{\mathrm{CbCr}}$. In this study, we focus on the 4:2:0 subsampling format, although our discussion is applicable to the $4: 2: 2$ format which subsamples the $(C b, C r)$-pair for each row of $B^{C b C r}$.

After subsampling the $\mathrm{CbCr}$ image $I^{C b C r}$, the subsampled $\mathrm{CbCr}$ image $I^{s u b, C b C r}$ is stored as a quarter-sized array, called the quarter-sized subsampled chroma image $I^{q, C b C r}$. Then, $I^{q, C b C r}$ and the luma image $I^{Y}$ are fed into the encoder for compression. After compressing $I^{q, C b C r}$ and $I^{Y}$, the compressed bitstring of $I^{q, C b C r}$ and $I^{Y}$ is sent to the receiver via a communication network, as depicted in Fig. 1

After decompressing $I^{q, C b C r}$ and $I^{Y}$ by the decoder, as depicted at the client side of Fig. 1, using the receiverselected chroma upsampling method, $I^{q, C b C r}$ is upsampled to obtain the upsampled chroma image $I^{u p, C b C r}$. Furthermore, $I^{u p, C b C r}$ and $I^{Y}$ are converted to the reconstructed RGB full-color image $I^{r e c, R G B}$ using the following YCbCr-to-RGB color conversion:

$$
\left[\begin{array}{l}
R_{i} \\
G_{i} \\
B_{i}
\end{array}\right]=\left[\begin{array}{ccc}
1.164 & 0 & 1.596 \\
1.164 & -0.391 & -0.813 \\
1.164 & 2.018 & 0
\end{array}\right]\left[\begin{array}{c}
Y_{i}-16 \\
C b_{i}-128 \\
C r_{i}-128
\end{array}\right]
$$

In our study, seven widely used chroma subsampling methods, namely 4:2:0(DIRECT), 4:2:0(L), 4:2:0(R), 4:2:0(A), IDID (interpolation dependent image downsampling) [19], JCDU (joint chroma downsampling and upsampling) [17], and Anchor [9], are considered, and they will be introduced in Subsection II.A. For simplicity, 4:2:0(DIRECT) is abbreviated as 4:2:0(D). It is notable that the Anchor method has been supported by the Versatile Video Coding (VVC) standard [15] and its predecessor High Efficiency Video Coding (HEVC) standard [2]. For convenience, let the set symbol $C_{s}$ denote the above seven chroma subsampling methods.

In chroma upsampling, the four commonly used chroma upsampling methods, namely NN (nearest neighbor), 4-tap, INTER_LINEAR [10], [11], and INTER_CUBIC [10], [11], are considered, and they will be introduced in Subsection III.A. It is notable that $\mathrm{NN}$ and 4-tap have been supported by VVC and HEVC; INTER_LINEAR and INTER_CUBIC have been supported by the OpenCV library. For convenience, let the set symbol $C_{u}$ denote the above four chroma upsampling methods. 


\section{A. Motivation}

For chroma subsampling and upsampling in the current coding system, as depicted in Fig. 1, it has one drawback in real scenarios. The drawback is that at the server side, only the chroma subsampling method "Anchor" is provided by VVC, and at the client side, only the two chroma upsampling methods, NN and 4-tap, are provided by VVC. The limited number of combinations, i.e. two combinations: Anchor-NN and Anchor-(4-tap), limiting number of combinations limits the quality of the reconstructed RGB full-color image.

Naturally, at the server side, besides the Anchor method, there are more chroma subsampling methods, e.g. those methods in $C_{s}$, that can be considered in the header of the encoder to offer more options. Similarly, at the client side, besides the NN and 4-tap methods, the two upsampling methods, namely INTER_LINEAR and INTER_CUBIC, can also be included to offer more options. However, among the 28 combinations in $C_{s} \mathrm{x} C_{u}$, many combinations may suffer from the coordinate displacement (CD) bias problem in the transition from the server side to the client side. The CD bias problem will be defined in Sections II-III, and we will explain why at the client side, the CD bias problem between the luma image and the upsampled chroma image degrades the quality of the reconstructed RGB full-color image.

To the best of our knowledge, in the literature, no methods were proposed for compensating the above-mentioned CD bias problem for combinations in $C_{s} \times C_{u}$. It first prompted us to develop a systematic way to analyze the CD bias problem for $C_{s} \times C_{u}$. Next, it prompted us to develop an effective CD compensation-based (CDC-based) method to eliminate the $\mathrm{CD}$ bias problem, enhancing the quality of the reconstructed RGB full-color image. Then, it also prompted us to propose an augmented coding system, as depicted in Fig. 2, where CD vector $(\mathrm{CDV})$ is used to measure the $\mathrm{CD}$ bias of the considered combination, to support the realization of the proposed CDCbased quality enhancement method.

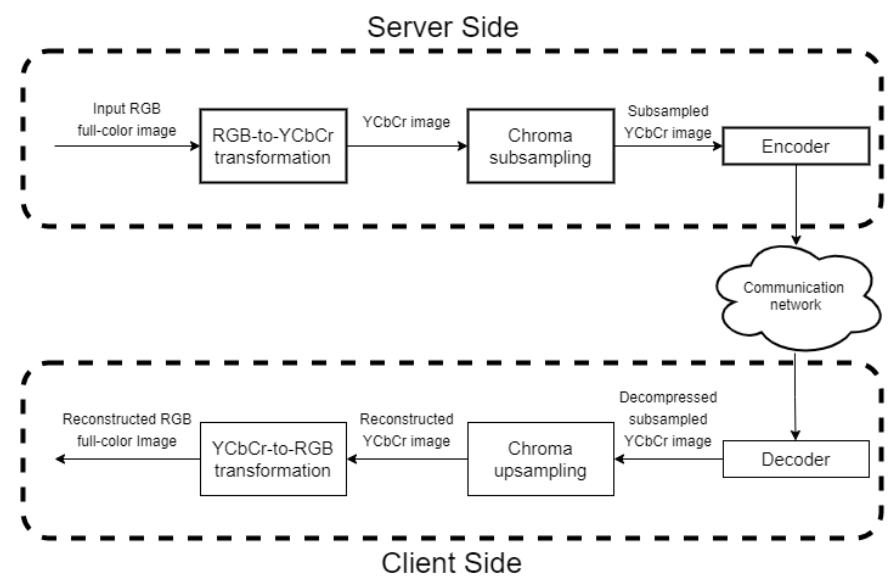

Fig. 1. The current coding system for compressing $I^{R G B}$.

\section{B. Contributions}

In this paper, the three contributions of the proposed CDCbased quality enhancement method for chroma subsampling and upsampling in our augmented coding system, as depicted in Fig. 2, are clarified as follows.

(1) At the server side, after performing one chroma subsampling method $c_{s}\left(\in C_{s}\right)$ on the current $2 \times 2 \mathrm{CbCr}$ block $B^{C b C r}$, we utilize the moment balance law[8] to analyze the subsampled chroma position of $B^{C b C r}$. Then, we analyze the $\mathrm{CD}$ bias problem occurring in the mapping from the subsampled $\mathrm{CbCr}$ image $I^{s u b, C b C r}$ to the quarter-sized subsampled chroma image $\mathrm{I}^{q, \mathrm{CbCr}}$ which will be fed into the encoder for compression. In our augmented coding system in Fig. 2, due to $\left|C_{s}\right|=7$, the sender can write a 3-bit codeword into the extended header of the compressed subsampled YCbC image to specify which chroma subsampling method is used at the server side.

(2) At the client side, for each combination " $c_{s}-c_{u}$ " in $C_{s} \mathrm{x} C_{u}$, we analyze the resultant $\mathrm{CD}$ bias problem occurring in the transition from the server side to the client side. For each combination, the resultant $\mathrm{CD}$ bias is measured by a CDV. Then, a built-in CDV table is built up to record the CDV information for all combinations in $C_{s} \mathrm{x} C_{u}$. Further, the builtin CDV table is plugged into the decoder of the proposed augmented coding system. According to the sender-selected chroma subsampling method " $c_{s}$ ", whose information can be extracted from the extended header of the compressed subsampled YCbC image, and the receiver-selected chroma upsampling method " $c_{u}$ ", the CDV information can be extracted from the built-in CDV table. Using the extracted CDV information, the proposed CDC-based chroma upsampling method, which consists of the receiver-selected chroma upsampling method and the proposed CDC-based quality enhancement method in Fig. 2, can be used to eliminate the $\mathrm{CD}$ bias side effect occurring in the combination " $c_{s}-c_{u}$ ", thus improving the quality of the reconstructed RGB full-color image.

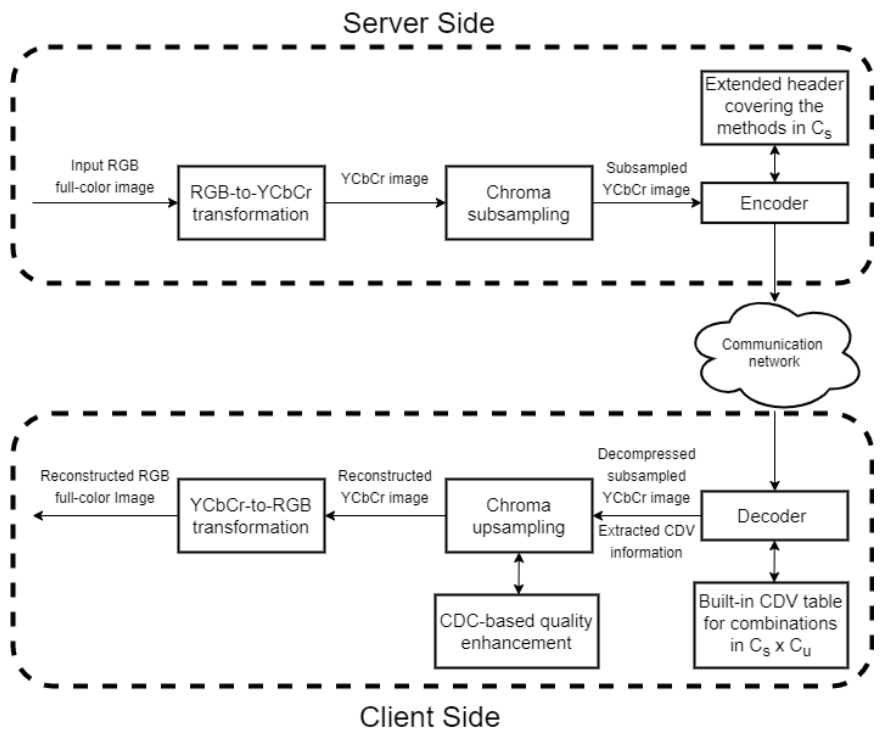

Fig. 2. The proposed augmented coding system for compressing $I^{R G B}$

(3) Based on the IMAX dataset with 18 images [4], the Kodak dataset with 24 images [5], the SCI (screen content images) dataset with 26 images [13], and the Video datasets with 200 images [16], the comprehensive experimental data 


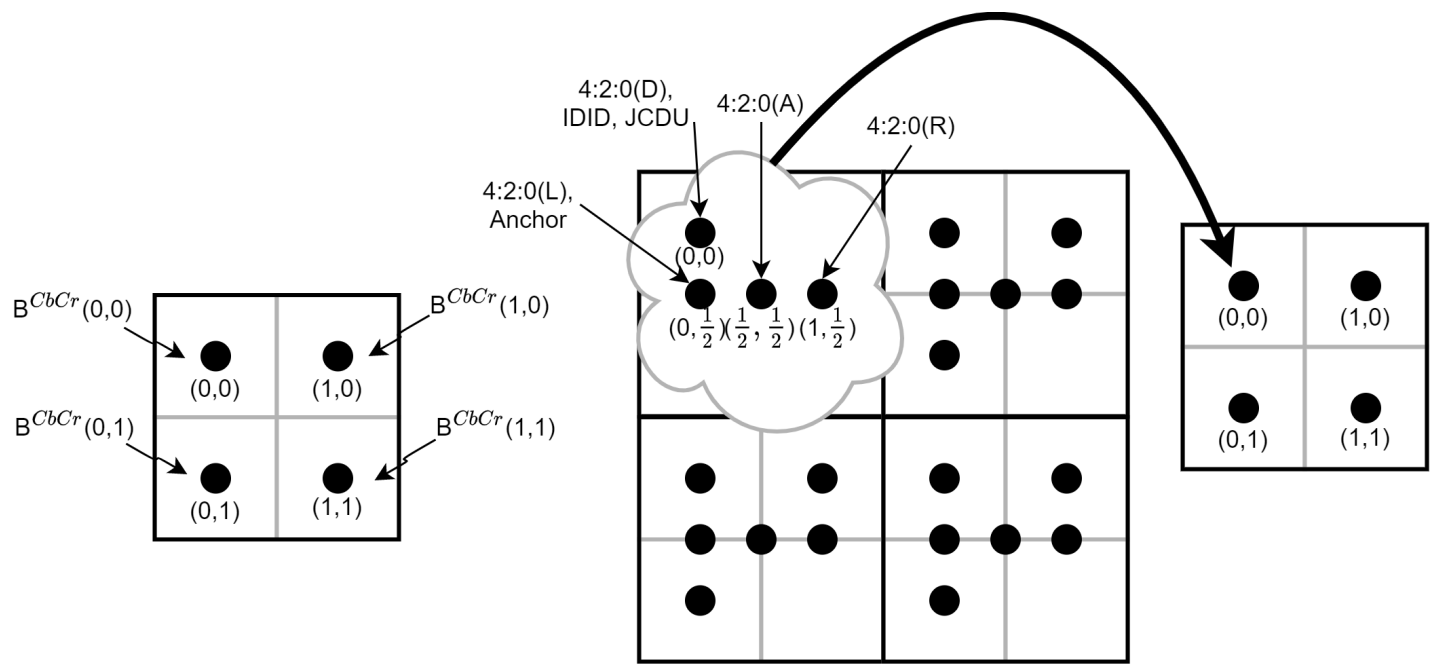

(a)

(b)

(c)

Fig. 3. The $\mathrm{CD}$ bias problem occurring in the mapping from $I^{s u b, C b C r}$ to $I^{q, C b C r}$. (a) The coordinates of the four entries in $B^{C b C r}$. (b) The subsampled chroma positions of the seven chroma subsampling methods in $C_{S}$. (c) The CD bias problem caused by mapping $I^{s u b, C b C r}$ to $I^{q, C b C r}$.

have demonstrated that on the newly released VVC platform VTM-12.0 [15], among the 28 combinations in $C_{s} \mathrm{x} C_{u}$, the proposed CDC-based quality enhancement method in our augmented coding system can achieve substantial quality and quality-bitrate tradeoff improvements for the 17 combinations. Here, the quality metrics used include CPSNR (color peak signal-to-noise ratio), SSIM (structure similarity index) [18], and visual effect. The quality-bitrate tradeoff metrics used include BD-rate (Bjøntegaard delta rate difference) [1], BD-CPSNR (Bjøntegaard delta CPSNR difference) [1], and the RD (rate-distortion) curves. Instead of the two $\mathrm{CD}$-free (coordinate displacement-free) VVC-supporting combinations, Anchor-NN and Anchor-(4-tap), the results of this paper can provide more CDC-based and CD-free combination options to meet user demands for higher quality of the reconstructed RGB full-color images.

The rest of this paper is organized as follows. In Section II, the $\mathrm{CD}$ bias problem occurring in the mapping from $I^{\mathrm{sub}, \mathrm{CbCr}}$ to $\mathrm{I}^{q, \mathrm{CbCr}}$ at the server side is analyzed. In addition, the extended header of the compressed subsampled $\mathrm{YCbC}$ image is highlighted. In Section III, the resultant CD bias problem occurring in each combination from the server side to the client side is analyzed. Next, the built-in CDV table is plugged into the decoder. In Section IV, the proposed CDC-based quality enhancement method is presented. In Section V, the thorough experimental results are illustrated to justify the substantial quality enhancement and quality-bitrate tradeoff merits of the proposed CDC-based quality enhancement method in the augmented coding system. In Section VI, some concluding remarks are offered.

To reduce the paper length, some symbols and abbreviations are frequently used and they are defined as follows:

$B^{C b C r}$ : the current $2 \times 2 \mathrm{CbCr}$ block with four entries, namely $B^{C b C r}(0,0), \quad B^{C b C r}(0,1), \quad B^{C b C r}(1,0), \quad$ and $B^{C b C r}(1,1)$.
$B_{l}^{C b C r}$ : the left neighboring $2 \times 2 \mathrm{CbCr}$ block of $B^{C b C r}$. $B_{u}^{C b C r}$ : the upper neighboring $2 \times 2 \mathrm{CbCr}$ block of $B^{C b C r}$. $B^{s u b, C b C r}$ : the subsampled chroma pair of $B^{C b C r}$.

$\mathrm{CD}$ : the abbreviation of "coordinate displacement".

CDC: the abbreviation of "coordinate displacement compensation".

CD-free: cause no CD bias problem.

CDV: the abbreviation of "coordinate displacement vector".

$c_{s}$ : the chroma subsampling method used.

$C_{s}$ : the set of the considered seven chroma subsampling methods and $\left|C_{s}\right|=7$.

$c_{u}$ : the chroma upsampling method used.

$c_{s}-c_{u}$ : one combination indicates that " $c_{s}$ " is used at the server side and " $c_{u}$ " is used at the client side.

$C_{u}$ : the set of the considered four chroma subsampling methods and $\left|C_{u}\right|=4$.

$C_{s} \times C_{u}$ : the set of all 28 combination, i.e. $\left\{c_{s}-c_{u} \mid c_{s} \in C_{s}\right.$ and $\left.c_{u} \in C_{u}\right\}$.

$I^{\mathrm{CbCr}}$ : the $\mathrm{CbCr}$ image.

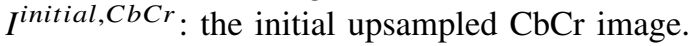

$I^{q, C b C r}$ : the quarter-sized subsampled chroma image prepared for compression.

$I^{s u b, C b C r}$ : the subsampled chroma image after performing one chroma subsampling method $c_{s}$ on $I^{C b C r}$.

$I^{u p, C b C r}$ : the upsampled $\mathrm{CbCr}$ image.

$I^{Y}$ : the luma image.

$\mathrm{S}_{C D}$ : the 17 combinations in $C_{s} \times C_{u}$, each combination with nonzero CDV.

\section{THE CD BIAS PROBLEM OCCURRING AT THE SERVER SIDE AND THE EXTENDED HEADER}

We first utilize the moment balance law [8] to determine the subsampled chroma position of $B^{C b C r}$, i.e. the position of $B^{s u b, C b C r}$, using the chroma subsampling method $c_{s} \in C_{s}$. Next, we analyze the $\mathrm{CD}$ bias problem occurring in the 
mapping from $I^{s u b, C b C r}$ to $I^{q, C b C r}$ at the server side. Then, the extended header design of the compressed subsampled $\mathrm{CbCr}$ image is highlighted.

\section{A. Using the Moment Balance Law to Determine the Subsam- pled Chroma Positions}

In this subsection, for one chroma subsampling method $c_{s}$ in $C_{S}$, we utilize the moment balance law [8] to determine the position of $B^{s u b, C b C r}$. Based on raster scanning order, the four entries of $B^{C b C r}$ are expressed as $B^{C b C r}(0,0), B^{C b C r}(1$, $0), B^{C b C r}(0,1)$, and $B^{C b C r}(1,1)$, as depicted in Fig. 3(a).

1) The subsampled chroma position of $B^{C b C r}$ using $4: 2: 0(D), 4: 2: O(L)$, and 4:2:O(R): 4:2:0(D) selects the entry $B^{C b C r}(0,0)$ as $B^{s u b, C b C r}$. Therefore, the position of $B^{s u b, C b C r}$ using 4:2:0(D) is located at $(0,0)$, as depicted in Fig. 3(b). 4:2:0(L) subsamples the chroma pair of $B^{C b C r}$ by averaging $B^{C b C r}(0,0)$ and $B^{C b C r}(0,1)$, and $B^{s u b, C b C r}$ equals $\left(\frac{1}{2} B^{C b C r}(0,0)+\frac{1}{2} B^{C b C r}(0,1)\right.$. Let the position of $B^{s u b, C b C r}$ using $4: 2: 0(\mathrm{~L})$ be located at $(0, \mathrm{y})$ where $(0, \mathrm{y})$ denotes the balance point between the two weights, namely $\frac{1}{2}$ and $\frac{1}{2}$, in the above expression of $B^{s u b, C b C r}$. According to the moment balance law, it yields

$$
\frac{1}{2} y=\frac{1}{2}(1-y)
$$

Solving Eq. (3), the solution equals $y=\frac{1}{2}$. On the other hand, the balance point equals $\left(0, \frac{1}{2}\right)$. Therefore, the position of $B^{s u b, C b C r}$ using 4:2:0(L) is located at $\left(0, \frac{1}{2}\right)$, as depicted in Fig. 3(b). Throughout this paper, the sentence "the position of $B^{s u b, C b C r}$ using the chroma subsampling method $c_{s}$ " and the sentence "the subsampled chroma position of $c_{s}$ " denote the same thing. By the same argument, the subsampled chroma position of 4:2:0(R) is located at $\left(1, \frac{1}{2}\right)$, as depicted in Fig. 3(b).

2) The position of $B^{s u b, C b C r}$ using 4:2:O(A) and Anchor [9]: 4:2:0(A) subsamples the chroma pair of $B^{C b C r}$ by averaging the four entries of $B^{C b C r}$, and it yields

$$
\begin{aligned}
B^{s u b, C b C r}= & \frac{1}{4}\left(B^{C b C r}(0,0)+B^{C b C r}(1,0)\right. \\
& \left.+B^{C b C r}(0,1)+B^{C b C r}(1,1)\right) \\
= & \frac{1}{2}\left(\frac{1}{2}\left(B^{C b C r}(0,0)+B^{C b C r}(0,1)\right)\right. \\
& \left.+\frac{1}{2}\left(B^{C b C r}(1,0)+B^{C b C r}(1,1)\right)\right)
\end{aligned}
$$

In Eq. (4), the subsampled chroma position of $\left(\frac{1}{2}\left(B^{C b C r}(0,0)+B^{C b C r}(0,1)\right)\right.$ is equal to that of 4:2:0(L), i.e. $\left(0, \frac{1}{2}\right)$. The subsampled chroma position of $\frac{1}{2}\left(B^{C b C r}(1,0)+B^{C b C r}(1,1)\right)$ is equal to that of $4: 2: 0(\mathrm{R})$, i.e. $\left(1, \frac{1}{2}\right)$.

Let $B^{a v g, C b C r}(0, *)=\frac{1}{2}\left(B^{C b C r}(0,0)\right)+B^{C b C r}(0,1)$ and $B^{a v g, C b C r}(1, *)=\frac{1}{2}\left(B^{C b C r}(1,0)+B^{C b C r}(1,1)\right)$. For $4: 2: 0(\mathrm{~A})$, it yields $B^{\text {sub, } C b C r}=\frac{1}{2} B^{a v g, C b C r}(0, *)+$ $\frac{1}{2} B^{a v g, C b C r}(1, *)$. Let the subsampled chroma position of 4:2:0(A) be denoted by $\left(x, \frac{1}{2}\right)$ where $\left(x, \frac{1}{2}\right)$ denotes the balance point between the two weights, namely $\frac{1}{2}$ and $\frac{1}{2}$, in the above expression of $B^{s u b, C b C r}$. According to the moment balance law, it yields

$$
\frac{1}{2} x=\frac{1}{2}(1-x)
$$

Solving Eq. (5), it yields $x=\frac{1}{2}$. Therefore, the position of $B^{s u b, C b C r}$ using $4: 2: 0(A)$ is located at $\left(0, \frac{1}{2}\right)$, as depicted in Fig. 3(b).

The Anchor method consists of two steps. In the first step, a 3-tap filter $[1,6,1] / 8$ performs two inner product operations, $<(1 / 8,6 / 8,1 / 8), \quad\left(B_{l}^{C b C r}(1,0), B^{C b C r}(0,0)\right.$, $\left.B^{C b C r}(1,0)\right)^{T}>\quad$ and $<(1 / 8, \quad 6 / 8, \quad 1 / 8), \quad\left(B_{l}^{C b C r}(1,1)\right.$, $\left.B^{C b C r}(0,1), B^{C b C r}(1,1)\right)^{T}>$, where $B_{l}^{C b C r}$ denotes the left neighboring $2 \times 2 \mathrm{CbCr}$ block of $B^{C b C r}$. Because the 3-tap filter used in the first step is symmetric, the balance point of the response of the first inner product operation is located at $(0,0)$, and the response of the inner product result is still saved in $B^{C b C r}(0,0)$. Similarly, the balance point of the response of the second inner product operation is located at $(0,1)$, so the response of the inner product result is still saved in $B^{C b C r}(0,1)$.

In the second step, the asymmetric 3-tap filter ( $[0,4$, $4] / 8)^{T}$ performs the inner product operation, $<(0 / 8,4 / 8,4 / 8)$, $\left(B_{u}^{C b C r}(0,1), B^{C b C r}(0,0), B^{C b C r}(0,1)\right)^{T}>$ where $\mathrm{B}_{u}^{C b C r}$ denotes the upper neighboring $2 \times 2 \mathrm{CbCr}$ block of $\mathrm{B}^{C b C r}$. After completing the second step, let $(0, y)$ denote the balance point of the response. According to the moment balance law, it yields

$$
\frac{0}{8}(1+y)+\frac{4}{8} y=\frac{4}{8}(1-y)
$$

Solving Eq. (6), it yields $y=\frac{1}{2}$. Therefore, the position of $B^{s u b, C b C r}$ using Anchor is located at $\left(0, \frac{1}{2}\right)$, as depicted in Fig. 3(b).

3) The subsampled chroma positions of $B^{C b C r}$ using IDID [19] and JCDU [17]: Zhang et al. [19] proposed an IDID chroma subsampling method using the new edge-directed interpolation [7]. For screen content images (SCIs) [22], Wang et al. [17] deployed the palette mode [12] in their JCDU chroma subsampling method. Each time, both methods consider a $16 \times 16$ chroma block in $I^{C b C r}$, and a $8 \times 8$ subsampled chroma block, in which each subsampled chroma pair is located at an integer coordinate, is generated. As a result, for each $2 \times 2$ chroma block in the considered $16 \times 16$ chroma block, the subsampled chroma positions of $\mathrm{B}^{\mathrm{CbCr}}$ using IDID and JCDU are all located at $(0,0)$, as depicted in Fig. 3(b).

\section{B. The Analysis of the CD Bias Problem at the Server Side}

In this subsection, we analyze the $\mathrm{CD}$ bias problem occurring in the mapping from $I^{s u b, C b C r}$ to $I^{q, C b C r}$ at the server side. As depicted in the mapping from Fig. 3(b) to Fig. 3(c), for compression, the subsampled chroma image $I^{s u b, C b C r}$ should be mapped to a quarter-sized subsampled chroma image $I^{q, C b C r}$. On the other hand, for each $2 \times 2 \mathrm{CbCr}$ block $B^{C b C r}$, the position of $B^{s u b, C b C r}$, namely (x, y), $0 \leq \mathrm{x}, \mathrm{y} \leq$ 1 , should be mapped to the relative location $(0,0)$ in $I^{q, C b C r}$. As a result, as depicted by the four-to-one mapping from Fig. 3(b) to Fig. 3(c), the four chroma subsampling methods, 


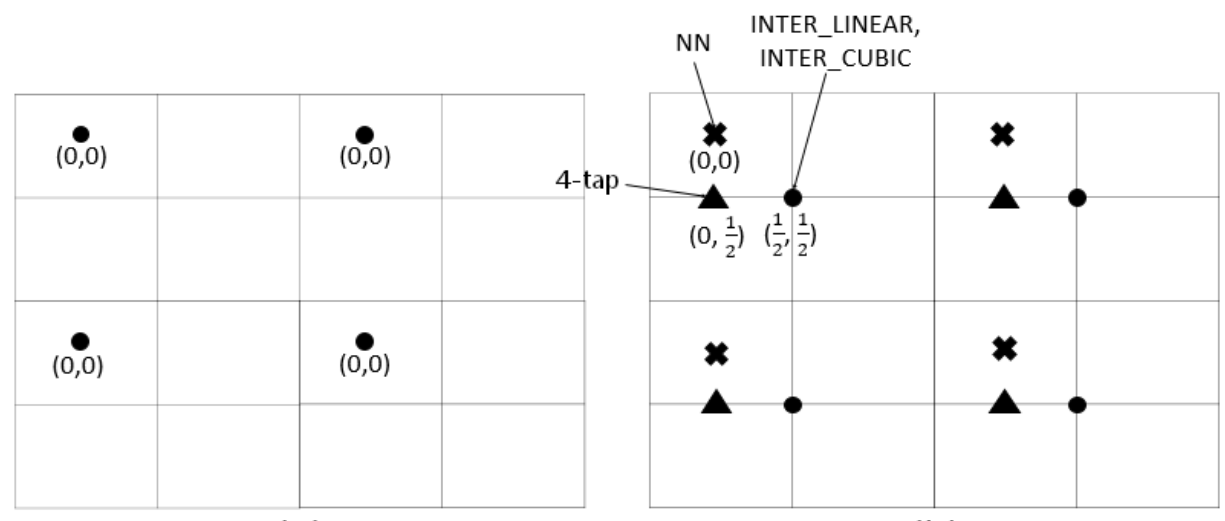

(a)

(b)

Fig. 4. The CD bias problem occurring in the $\left(\delta_{x}, \delta_{y}\right)$-moving operation of chroma upsampling at the client side. (a) The initial upsampled chroma pixel $I^{\text {initial, } \mathrm{CbCr}}(x, y)$. (b) After $\left(\delta_{x}, \delta_{y}\right)$-moving operation, the shifted upsampled chroma pixel $I^{\text {initial }, C b C r}\left(\mathrm{x}+\delta_{x}, \mathrm{y}+\delta_{y}\right)$.

4:2:0(A), 4:2:0(R), 4:2:0(L), and Anchor, cause different CD bias problems.

The above $\mathrm{CD}$ bias problem occurring in one chroma subsampling method " $c_{s}$ " can be measured as a CDV, namely $(\Delta x, \Delta y)$, where $\Delta x=0-x$ and $\Delta y=0-y$. Table I lists the CDV of each chroma subsampling method in $C_{S}$, and we observe that for 4:2:0(A), 4:2:0(L), 4:2:0(R), and Anchor, the CD bias, in terms of CDVs, have been embedded into $I^{q, C b C r}$. Furthermore, $I^{q, C b C r}$ and the luma image $I^{Y}$ are fed into the encoder for compression. Because at the server side, we never do subsampling on $I^{Y}$, it thus causes a CD bias problem between $I^{Y}$ and $I^{q, C b C r}$. As depicted in Fig. 2, the sender can write a 3-bit codeword into the extended header of the compressed subsampled $\mathrm{YCbC}$ image to specify which chroma subsampling method " $c_{s}$ " is used at the server side. At the client side, the receiver can extract the information of the chroma subsampling method " $c_{s}$ " used at the server side from the extended header.

TABLE I

THE CDV OF EACH CHROMA SUBSAMPLING METHOD.

\begin{tabular}{|c|c|c|c|c|}
\hline $\begin{array}{c}\text { chroma } \\
\text { subsampling method }\end{array}$ & $\begin{array}{c}\text { subsampled } \\
\text { chroma position }\end{array}$ & mapped position & CDV & CD bias problem \\
\hline $4: 2: 0(\mathrm{~A})$ & $\left(\frac{1}{2}, \frac{1}{2}\right)$ & $(0,0)$ & $\left(-\frac{1}{2},-\frac{1}{2}\right)$ & YES \\
\hline $4: 2: 0(\mathrm{~L})$ & $\left(0, \frac{1}{2}\right)$ & $(0,0)$ & $\left(0,-\frac{1}{2}\right)$ & YES \\
\hline $4: 2: 0(\mathrm{R})$ & $\left(1, \frac{1}{2}\right)$ & $(0,0)$ & $\left(-1,-\frac{1}{2}\right)$ & YES \\
\hline $4: 2: 0(\mathrm{D})$ & $(0,0)$ & $(0,0)$ & $(0,0)$ & NO \\
\hline Anchor & $\left(0, \frac{1}{2}\right)$ & $(0,0)$ & $\left(0,-\frac{1}{2}\right)$ & YES \\
\hline IDID [19] & $(0,0)$ & $(0,0)$ & $(0,0)$ & NO \\
\hline JCDU [17] & $(0,0)$ & $(0,0)$ & $(0,0)$ & NO \\
\hline
\end{tabular}

\section{THE CD BIAS PROBLEM ANALYSIS FOR COMBINATIONS AND THE BUILT-IN CDV TABLE}

In this section, for each combination in $C_{s} \times C_{u}$, we first analyze the $\mathrm{CD}$ bias problem for only chroma upsampling at the client side. Next, we analyze the resultant CD bias problem occurring in the transition from the server side to the client side. Then, a built-in CDV table is plugged in the decoder, and the built-in CDV table further serves as the base of our CDCbased quality enhancement method which will be presented in the next section.

\section{A. The CD Bias Problem Analysis for only Chroma Upsam- pling at the Client Side}

After receiving the decompressed quarter-sized subsampled chroma image $I^{q, C b C r}$ from the decoder, the receiver-selected chroma upsampling method " $c_{u}$ " $\left(\in C_{u}\right)$ first moves each pixel $I^{q, C b C r}(x, y)$, as marked by a black bullet in Fig. 3(c), to $I^{u p, C b C r}(2 x, 2 y)$, as marked by a black bullet in Fig. 4(a), to produce the initial upsampled chroma image $I^{\text {initial, } \mathrm{CbCr}}$.

TABLE II

THE CDV OF EACH CHROMA UPSAMPLING METHOD.

\begin{tabular}{|c|c|c|c|c|}
\hline $\begin{array}{c}\text { chroma } \\
\text { upsampling method }\end{array}$ & $\begin{array}{c}\text { initial subsampled } \\
\text { chroma position }\end{array}$ & mapped position & CDV & CD bias problem \\
\hline INTER_LINEAR & $(0,0)$ & $\left(\frac{1}{2}, \frac{1}{2}\right)$ & $\left(\frac{1}{2}, \frac{1}{2}\right)$ & YES \\
\hline INTER_CUBIC & $(0,0)$ & $\left(\frac{1}{2}, \frac{1}{2}\right)$ & $\left(\frac{1}{2}, \frac{1}{2}\right)$ & YES \\
\hline NN & $(0,0)$ & $(0,0)$ & $(0,0)$ & NO \\
\hline 4-tap & $(0,0)$ & $\left(0, \frac{1}{2}\right)$ & $\left(0, \frac{1}{2}\right)$ & YES \\
\hline
\end{tabular}

1) The CD bias problem for INTER_LINEAR and INTER_CUBIC: When applying the chroma upsampling method "INTER_LINEAR" (or "INTER_CUBIC") to upsample $I^{\text {initial }, \mathrm{CbCr}}$, each pixel $I^{\text {initial }, \mathrm{CbCr}}(2 i, 2 j)$ is first moved to $I^{\text {initial, } \mathrm{CbCr}}\left(2 x+\frac{1}{2}, 2 y+\frac{1}{2}\right)$ [10], [11], as marked by a black bullet in Fig. 4(b). The above $\left(\frac{1}{2}, \frac{1}{2}\right)$-moving operation yields $\mathrm{CDV}=\left(\frac{1}{2}, \frac{1}{2}\right)\left(=\left(\frac{1}{2}, \frac{1}{2}\right)-(0,0)\right)$, as listed in Table

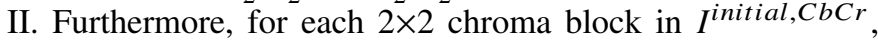
based on the window-based approach, INTER_LINEAR (or INTER_CUBIC) fills the four missing chroma pixels in the $2 \times 2$ chroma block.

2) The CD bias problem for $N N$ : When applying the chroma upsampling method "NN" to upsample $I^{\text {initial }, C b C r}$, it is unnecessary to move $I^{\text {initial, } C b C r}(2 x, 2 y)$, as marked by a cross symbol in Fig. 4(b), to somewhere else. It yields CDV $=(0,0)$, as listed in Table II. Furthermore, based on the nearest neighbor approach, for each $2 \times 2$ chroma block in 
TABLE III

THE CDV OF EACH COMBINATION.

\begin{tabular}{|c|c|c|c|c|c|}
\hline $\begin{array}{c}\text { chroma } \\
\text { subsampling method }\end{array}$ & $\begin{array}{c}\text { chroma } \\
\text { upsampling method }\end{array}$ & $\begin{array}{l}\text { CDV at the } \\
\text { server side }\end{array}$ & $\begin{array}{l}\text { CDV at the } \\
\text { client side }\end{array}$ & $\begin{array}{l}\text { CDV of each } \\
\text { combination }\end{array}$ & $\mathrm{CD}$ bias problem \\
\hline \multirow{4}{*}{$4: 2: 0(\mathrm{~A})$} & INTER_LINEAR & \multirow{4}{*}{$\left(-\frac{1}{2},-\frac{1}{2}\right)$} & $\left(\frac{1}{2}, \frac{1}{2}\right)$ & $(0,0)$ & No \\
\hline & INTER_CUBIC & & $\left(\frac{1}{2}, \frac{1}{2}\right)$ & $(0,0)$ & No \\
\hline & NN & & $(0,0)$ & $(0,0)$ & No \\
\hline & 4-tap & & $\left(0, \frac{1}{2}\right)$ & $\left(-\frac{1}{2}, 0\right)$ & YES \\
\hline \multirow{4}{*}{$4: 2: 0(\mathrm{~L})$} & INTER_LINEAR & \multirow{4}{*}{$\left(0,-\frac{1}{2}\right)$} & $\left(\frac{1}{2}, \frac{1}{2}\right)$ & $\left(\frac{1}{2}, 0\right)$ & YES \\
\hline & INTER_CUBIC & & $\left(\frac{1}{2}, \frac{1}{2}\right)$ & $\left(\frac{1}{2}, 0\right)$ & YES \\
\hline & $\overline{\mathrm{NN}}$ & & $(0,0)$ & $(0,0)$ & No \\
\hline & 4-tap & & $\left(0, \frac{1}{2}\right)$ & $(0,0)$ & No \\
\hline \multirow{4}{*}{$4: 2: 0(\mathrm{R})$} & INTER_LINEAR & \multirow{4}{*}{$\left(-1,-\frac{1}{2}\right)$} & $\left(\frac{1}{2}, \frac{1}{2}\right)$ & $\left(-\frac{1}{2}, 0\right)$ & YES \\
\hline & INTER_CUBIC & & $\left(\frac{1}{2}, \frac{1}{2}\right)$ & $\left(-\frac{1}{2}, 0\right)$ & YES \\
\hline & $\mathrm{NN}$ & & $(0,0)$ & $(0,0)$ & No \\
\hline & 4-tap & & $\left(0, \frac{1}{2}\right)$ & $(-1,0)$ & YES \\
\hline \multirow{4}{*}{$4: 2: 0(\mathrm{D})$} & INTER_LINEAR & \multirow{4}{*}{$(0,0)$} & $\left(\frac{1}{2}, \frac{1}{2}\right)$ & $\left(\frac{1}{2}, \frac{1}{2}\right)$ & YES \\
\hline & INTER_CUBIC & & $\left(\frac{1}{2}, \frac{1}{2}\right)$ & $\left(\frac{1}{2}, \frac{1}{2}\right)$ & YES \\
\hline & $\overline{\mathrm{NN}}$ & & $(0,0)$ & $(0,0)$ & No \\
\hline & 4-tap & & $\left(0, \frac{1}{2}\right)$ & $\left(0, \frac{1}{2}\right)$ & YES \\
\hline \multirow{4}{*}{ Anchor } & INTER_LINEAR & \multirow{4}{*}{$\left(0,-\frac{1}{2}\right)$} & $\left(\frac{1}{2}, \frac{1}{2}\right)$ & $\left(\frac{1}{2}, 0\right)$ & YES \\
\hline & INTER_CUBIC & & $\left(\frac{1}{2}, \frac{1}{2}\right)$ & $\left(\frac{1}{2}, 0\right)$ & YES \\
\hline & NN & & $(0,0)$ & $(0,0)$ & No \\
\hline & 4-tap & & $\left(0, \frac{1}{2}\right)$ & $(0,0)$ & No \\
\hline \multirow{4}{*}{ IDID [19] } & INTER_LINEAR & \multirow{4}{*}{$(0,0)$} & $\left(\frac{1}{2}, \frac{1}{2}\right)$ & $\left(\frac{1}{2}, \frac{1}{2}\right)$ & YES \\
\hline & INTER_CUBIC & & $\left(\frac{1}{2}, \frac{1}{2}\right)$ & $\left(\frac{1}{2}, \frac{1}{2}\right)$ & YES \\
\hline & NN & & $(0,0)$ & $(0,0)$ & No \\
\hline & 4-tap & & $\left(0, \frac{1}{2}\right)$ & $\left(0, \frac{1}{2}\right)$ & YES \\
\hline \multirow{4}{*}{ JCDU [17] } & INTER_LINEAR & \multirow{4}{*}{$(0,0)$} & $\left(\frac{1}{2}, \frac{1}{2}\right)$ & $\left(\frac{1}{2}, \frac{1}{2}\right)$ & YES \\
\hline & INTER_CUBIC & & $\left(\frac{1}{2}, \frac{1}{2}\right)$ & $\left(\frac{1}{2}, \frac{1}{2}\right)$ & YES \\
\hline & $\mathrm{NN}$ & & $(0,0)$ & $(0,0)$ & No \\
\hline & 4-tap & & $\left(0, \frac{1}{2}\right)$ & $\left(0, \frac{1}{2}\right)$ & YES \\
\hline
\end{tabular}

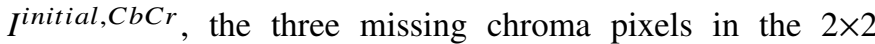
chroma block just copy the top-left chroma pixel of their own $2 \times 2$ chroma block.

3) The CD bias problem for 4-tap: For the chroma upsampling method "4-tap", according to the moment balance law, as proved in Appendix A, the 4-tap method first moves each pixel $I^{\text {initial }, C b C r}(2 x, 2 y)$ to $I^{\text {initial }, C b C r}\left(2 x, 2 y+\frac{1}{2}\right)$, as marked by a triangle symbol in Fig. 4(b). The above $\left(0, \frac{1}{2}\right)$ moving operation yields CDV $=\left(0, \frac{1}{2}\right)$, as listed in Table II. Furthermore, based on the 4-tap filter operation, as described in Appendix A, for each $2 \times 2$ chroma block in $I^{\text {initial, } C b C r}$, the four missing pixels can be reconstructed.

\section{B. The CD Bias Problem Analysis for Combinations from the Server Side to the Client Side}

We analyze the $\mathrm{CD}$ bias problem for all combinations in $C_{s} \times C_{u}$. For each combination, its $\mathrm{CD}$ bias problem is also expressed as a CDV. For one CD-free combination, its CDV is a zero vector; otherwise, its $\mathrm{CDV}$ is a nonzero vector. Furthermore, a built-in CDV table is constructed to record the CDV for each combination. The built-in table is plugged into the decoder and further serves as the base of the proposed CDC-based quality enhancement method.

1) The CD bias problem analysis for all combinations: For easy understanding, we analyze the $\mathrm{CD}$ bias problem for the four combinations, namely 4:2:0(A)-INTER_LINEAR,
4:2:0(L)-INTER_LINEAR, $c_{s}$-NN $\left(c_{s} \in C_{s}\right)$, and Anchor-(4tap), in detail.

1.1) The CD bias problem analysis for 4:2:0(A)INTER_LINEAR and 4:2:O(L)-INTER_LINEAR: From Table I, we know that at the server side, the chroma subsampling method "4:2:0(A)" causes a CD bias problem with $\mathrm{CDV}=$ $\left(-\frac{1}{2},-\frac{1}{2}\right)$. Table II indicates that at the client side, the chroma upsampling method "INTER_LINEAR" causes a CD bias problem with $\mathrm{CDV}=\left(\frac{1}{2}, \frac{1}{2}\right)$. For the combination "4:2:0(A)INTER_LINEAR", the resultant CDV equals the sum of the CDV (= $\left.\left(-\frac{1}{2},-\frac{1}{2}\right)\right)$ caused by " $4: 2: 0(\mathrm{~A})$ " and the CDV (= $\left.\left(\frac{1}{2}, \frac{1}{2}\right)\right)$ caused by "INTER_LINEAR". Therefore, the resultant CDV of the combination "4:2:0(A)-INTER_LINEAR" equals $(0,0)\left(=\left(-\frac{1}{2},-\frac{1}{2}\right)+\left(\frac{1}{2}, \frac{1}{2}\right)\right)$, as depicted in Table III, and it indicates that the combination "4:2:0(A)-INTER_LINEAR" is CD-free.

By the same argument, for the combination 4:2:0(L)INTER_LINEAR, the resultant CDV equals the sum of the CDV $\left(=\left(0,-\frac{1}{2}\right)\right)$ caused by "4:2:0(L)" and the CDV $\left(=\left(\frac{1}{2}\right.\right.$ ,$\left.\frac{1}{2}\right)$ ) caused by "INTER_LINEAR". Therefore, the resultant CDV of "4:2:0(L)-INTER_LINEAR" equals $\left(\frac{1}{2}, 0\right)\left(=\left(0,-\frac{1}{2}\right)\right.$ $\left.+\left(\frac{1}{2}, \frac{1}{2}\right)\right)$, as depicted in Table III, and it indicates that there is a CD bias problem occurring in the combination "4:2:0(L)INTER_LINEAR".

1.2) The $C D$ bias problem analysis for $c_{s}-N N\left(c_{s} \in C_{s}\right)$ and Anchor-(4-tap): In Table II, we know that at the client side, 


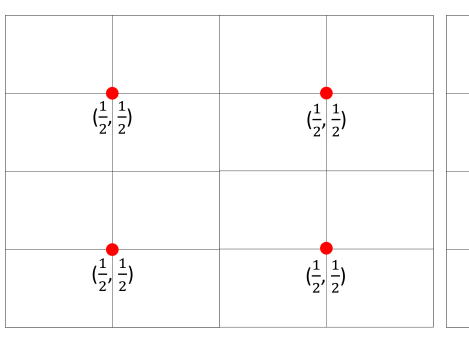

(a)

\begin{tabular}{|c|c|c|c|}
\hline$(0,0)$ & & $(0,0)$ & \\
\hline & & & \\
\hline 00,0$)$ & & $(0,0)$ & \\
\hline & & & \\
\hline & & & \\
\hline
\end{tabular}

(b)

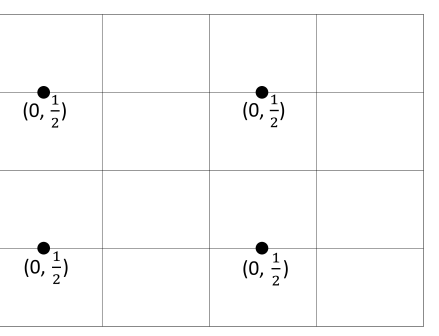

(c)

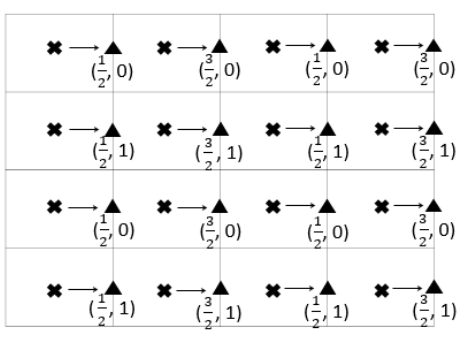

(d)

Fig. 5. The sketch of the proposed CDC-based quality enhancement method for the combination 4:2:0(A)-(4-tap). (a) The subsampled chroma image $I^{\text {sub }, \mathrm{C} b C r}$. (b) The initial upsampled chroma image $I^{\text {initial }, C b C r}$. (c) Moving each subsampled chroma pair of $I^{\text {initial, }}$, $b C r$ half a pixel downward, as marked by bullets. (d) The upsampled chroma image $I^{u p, C b C r}$ marked by the cross symbols. The CDC-based upsampled chroma image $I^{\text {enhanced, } \mathrm{CbCr}}$ marked by the triangle symbols.

the DVC of the chroma upsampling method "NN" is $(0,0)$. For each $2 \times 2$ chroma block, as depicted by one cross-marked pixel and three missing pixels in Fig. 4(b), the three missing chroma pixels in the block just copy the top-left chroma pixel of their own $2 \times 2$ block. Therefore, from the server side to the client side, the combination " $c_{s}-\mathrm{NN}$ " $\left(c_{s} \in C_{s}\right)$ is always CDfree no matter what chroma subsampling method " $c_{s}$ " is used because the copy-based upsampling method used in "NN" always eliminates the CDV caused in chroma subsampling. In Table III, the CDV of the combination $c_{s}-\mathrm{NN}$ is thus set to $(0,0)\left(=\left(0,-\frac{1}{2}\right)+\left(0, \frac{1}{2}\right)\right)$.

By the same argument, from the server side to the client side, the combination "Anchor-(4-tap)" also causes no CD bias problem because the sum of the CDV caused by "Anchor" at the server side and that caused by "4-tap" at the client side equals $(0,0)\left(=\left(0,-\frac{1}{2}\right)+\left(0, \frac{1}{2}\right)\right)$.

2) The built-in $C D V$ table stored in the decoder: As a result, the CDVs of all 28 combinations in $C_{s} \times C_{u}$ are listed in the built-in CDV table, i.e. Table III. The built-in CDV table is plugged into the decoder, as shown in the augmented coding system in Fig. 2. From Table III, we observe that due to deploying our quality enhancement method in each original combination with nonzero CDV, the related 17 combinations in $\mathrm{S}_{C D}$ become $\mathrm{CD}$-free, indicating the importance and practicality of this study.

In the next section, for any combination " $c_{s}-c_{u}$ " in $S_{C D}$, we propose an effective CDC-based chroma upsampling method, which consists of the receiver-selected chroma upsampling method " $c_{s}$ " and the proposed CDC-based quality enhancement method, to improve the quality of the reconstructed RGB full-color image.

\section{THE PROPOSED CDC-BASED QUALITY ENHANCEMENT METHOD}

As listed in Table III, for any combination in the set $S_{C D}$, there is a nonzero CDV existing in the upsampled chroma image $I^{u p, C b C r}$ at the client side, while the corresponding luma image $I^{Y}$ is $\mathrm{CD}$-free. Therefore, the $\mathrm{CD}$ bias problem between $I^{u p, C b C r}$ and $I^{Y}$ leads to the degradation of the reconstructed RGB full-color image.

We now propose a CDC-based quality enhancement method to eliminate the $\mathrm{CD}$ bias side effect in $I^{u p, C b C r}$, which was obtained using the one combination in $S_{C D}$, for improving the quality of the reconstructed RGB full-color image. From Table III, it is known that the five nonzero CDVs of the 17 combinations in $S_{C D}$ are $\left(-\frac{1}{2}, 0\right),\left(\frac{1}{2}, 0\right),(-1,0),\left(\frac{1}{2}, \frac{1}{2}\right)$, and $(0$, $\left.\frac{1}{2}\right)$. For the sake of understanding, in what follows, we take the combination 4:2:0(A)-(4-tap), whose CDV equals $\left(-\frac{1}{2}, 0\right)$, to explain how the proposed CDC-based quality enhancement method works in the augmented coding system in detail.

After performing 4:2:0(A) on the chroma image, the subsampled chroma image $I^{s u b, C b C r}$ is depicted in Fig. 5(a), where the subsampled chroma pairs are marked by the red bullets. Next, $I^{s u b, C b C r}$ is mapped to the quarter-sized subsampled chroma image $I^{q, C b C r}$ in Fig. 3(b), and the sender writes a 3-bit codeword into the header to specify that the chroma subsampling method "4:2:0(A)" is used. Then, $I^{q, C b C r}$ and $I^{Y}$ are fed into the encoder for compression.

After receiving the decompressed quarter-sized subsampled chroma image $I^{q, C b C r}$ by the receiver at the client side, the receiver-selected chroma upsampling method, say "4-tap", first constructs the initial upsampled chroma image $I^{\text {initial, } \mathrm{CbCr} \text {, as }}$ depicted in Fig. 5(b). Next, the 4-tap method moves each sub-

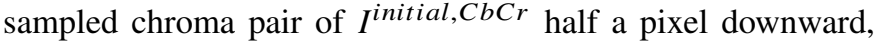
and the moved subsampled chroma pixels are marked by the black bullets in Fig. 5(c). Then the 4-tap method reconstructs all missing chroma pixels to obtain the upsampled chroma image $I^{u p, C b C r}$, in which the constructed chroma pixels are marked by the cross symbols in Fig. 5(d). From the built-in CDV table (see Table III) in the decoder, the CDV information of the combination "4:2:0(A)-(4-tap)" can be extracted and we have $\mathrm{CDV}=\left(-\frac{1}{2}, 0\right)$.

In order to eliminate the $\mathrm{CD}$ bias, i.e. $\mathrm{CDV}=\left(-\frac{1}{2}, 0\right)$, caused in "4:2:0(A)-(4-tap)", the proposed CDC-based quality enhancement method aims to replace each temporarily reconstructed chroma pixel, $I^{u p, C b C r}(x, y)$, as marked by the cross symbol in Fig. 5(d), by the newly enhanced chroma pixel in $I^{\text {enhanced, } C b C r}\left(\mathrm{x}+\frac{1}{2}, \mathrm{y}\right)$, as marked by the triangle symbol in Fig. 5(d). It is notable that the interpolation of the triangle-marked chroma pixel in Fig. 5(d) can be realized by averaging the two horizontally neighboring cross-marked chroma pixels. Finally, the CDC-based enhanced chroma image $I^{\text {enhanced }}, \mathrm{CbCr}$ is moved a half pixel to the left direction to align the luma image for enhancing the quality of the reconstructed RGB full-color image.

After discussing our quality enhancement method to elim- 


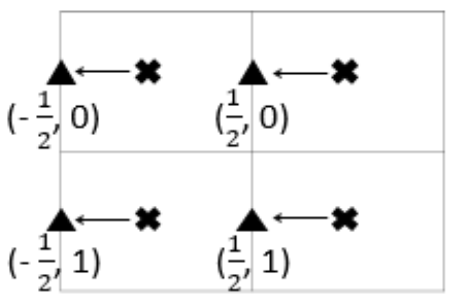

(a)

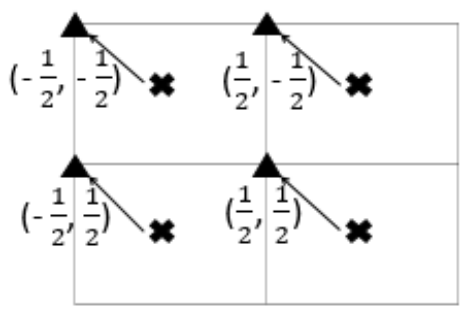

(c)

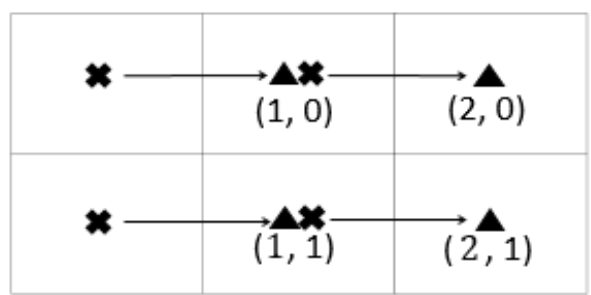

(b)

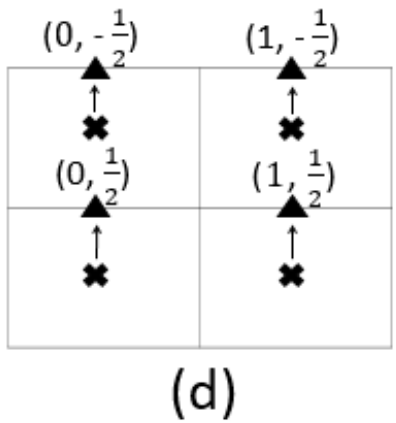

Fig. 6. The enhanced upsampled chroma image $I^{\text {enhanced, }} \mathrm{CbCr}$ using the proposed CDC-based quality enhancement method for the other four nonzero CDVs. (a) The enhanced chroma image for the combination with $\mathrm{CDV}=\left(\frac{1}{2}, 0\right)$. (b) The enhanced chroma image for the combination with $\mathrm{CDV}=(-1,0)$. (c) The enhanced chroma image for the combination with $\mathrm{CDV}=\left(\frac{1}{2}, \frac{1}{2}\right)(\mathrm{d})$ The enhanced chroma image for the combination with $\mathrm{CDV}=\left(0, \frac{1}{2}\right)$.

inate the $\mathrm{CD}$ bias problem occurring in the combination "4:2:0(A)-(4-tap)", for the other four nonzero CDVs in the set $S_{C D}$, namely $\left(\frac{1}{2}, 0\right),(-1,0),\left(\frac{1}{2}, \frac{1}{2}\right)$, and $\left(0, \frac{1}{2}\right)$, we replace each temporarily reconstructed chroma pixel, marked by the cross symbol in Figs. 6(a)-(d), with the newly enhanced chroma pixel, which is marked by the triangle symbol located at ( $\mathrm{x}$ $\left.\frac{1}{2}, \mathrm{y}\right),(\mathrm{x}+1, \mathrm{y}),\left(\mathrm{x}-\frac{1}{2}, \mathrm{y}-\frac{1}{2}\right),\left(\mathrm{x}, \mathrm{y}-\frac{1}{2}\right)$, respectively. It is notable that the re-interpolation of the triangle-marked chroma pixels in Fig. 6(a), Fig. 6(b), and Fig. 6(d) can be realized using a linear interpolation on the neighboring cross-marked chroma pixels, while the re-interpolation of the trianglemarked chroma pixels in Fig. 6(c) can be realized using a bilinear interpolation on the neighboring cross-marked chroma pixels. Finally, the enhanced chroma image is appropriately moved to align the luma image for enhancing the quality of the RGB full-color image.

\section{EXPERIMENTAL RESUlTS}

In this section, based on the IMAX, Kodak, Video, and SCI datasets, the comprehensive experimental data are demonstrated to show the quality and quality-bitrate tradeoff merits of our CDC-based quality enhancement method for the 17 combinations in $S_{C D}$. In addition, the execution time overhead of our quality enhancement method is also provided.

All the concerned combinations are implemented on a computer with an Intel Core i7-7700 CPU 3.6 GHz and $24 \mathrm{~GB}$ RAM. The operating system is the Microsoft Windows 1064 bit operating system. The program development environment is Visual C++ 2017.

\section{A. Quality Enhancement Merit and Time Comparison}

When setting QP (quantization parameter) to zero, we adopt the three quality metrics, namely CPSNR (color peak signal-to-noise ratio), PSNR, and SSIMc (structure similarity index) [18], to demonstrate the quality enhancement merit of our CDC-based quality enhancement method for the 17 combinations in $S_{C D}$.

CPSNR is used to evaluate the average quality of the reconstructed RGB full-color images for one dataset with $\mathrm{N}$ images, and it is defined by

$$
\mathrm{CPSNR}=\frac{1}{N} \sum_{n=1}^{N} 10 \log _{10} \frac{255^{2}}{C M S E}
$$

with $C M S E=\frac{1}{3 W H} \sum_{p \in P} \sum_{c \in\{R, G, B\}}\left[I_{n, c}^{R G B}(p)-\right.$ $\left.I_{n, c}^{r e c, R G B}(p)\right]^{2}$ in which $\mathrm{P}=\{(x, y) \mid 1 \leq x \leq H, 1 \leq y \leq W\}$ denotes the set of pixel coordinates in one $W \times H$ image. Here, $\mathrm{N}=18, \mathrm{~N}=24, \mathrm{~N}=200$, and $\mathrm{N}=20$ for the IMAX, Kodak, Video, and SCI datasets, respectively. $I_{n, c}^{R G B}(p)$ and $I_{n, c}^{r e c}, R G B(p)$ denote the c-color value of the pixel at position $p$ in the $n$th original RGB full-color image and the reconstructed image, respectively. For fairness, each image in the Kodak dataset is downsampled to a quarter-sized one such that the average size of the downsampled images is close to the average size of the downsampled images in the IMAX dataset. The average CPSNR value equals the mean of the four CPSNR values for the four datasets. The SSIMc value is measured by the mean of the three SSIM values for the R, G, and B color planes.

Table IV lists the quality and time performance for the 11 CD-free combinations in $C_{s} \times C_{u}-S_{C D}$. To demonstrate the 
TABLE IV

THE QUALITY AND TIME PERFORMANCE OF THE 11 COMBINATIONS IN $C_{s} \times C_{u}-S_{C D}$.

\begin{tabular}{|c|c|c|c|c|}
\hline \multicolumn{2}{|c|}{ Combination } & CPSNR & SSIMc & Time \\
\hline \multirow{3}{*}{$4: 2: 0(\mathrm{~A})$} & NN & 40.9286 & 0.9809 & 0.0083 \\
\cline { 2 - 5 } & INTER_LINEAR & 41.9151 & 0.9827 & 0.0084 \\
\cline { 2 - 5 } & INTER_CUBIC & 42.9122 & 0.9848 & 0.0081 \\
\hline \multirow{2}{*}{$4: 2: 0(\mathrm{~L})$} & $\mathrm{NN}$ & 39.6447 & 0.9771 & 0.0071 \\
\cline { 2 - 5 } & $4-$ tap & 41.4947 & 0.9810 & 0.0180 \\
\hline \multirow{2}{*}{$4: 2: 0(\mathrm{R})$} & $\mathrm{NN}$ & 39.5964 & 0.9769 & 0.0079 \\
\hline 4:2:0(D) & NN & 38.3516 & 0.9726 & 0.0073 \\
\hline IDID [19] & NN & 38.2285 & 0.9706 & 10.7912 \\
\hline JCDU [17] & NN & 38.8632 & 0.9730 & 0.1360 \\
\hline \multirow{2}{*}{ Anchor } & NN & 39.3730 & 0.9781 & 0.0081 \\
\cline { 2 - 5 } & $4-t a p$ & 39.5840 & 0.9816 & 0.0198 \\
\hline
\end{tabular}

quality merit of our quality enhancement method for the 17 combinations in $S_{C D}$, Table V lists the CPSNR and $\operatorname{SSIM}_{c}$ gains of our quality enhancement method, although little execution time overhead is required. In Table V, the average quality performance and the execution time performance of the reconstructed RGB full-color images using our method for each combination are tabulated in the parenthesis '()'. For example, for the combination "JCDU-INTER_CUBIC", the CPSNR gain of our CDC-based quality enhancement method can achieve $2.7657(=43.2027-40.4370) \mathrm{dB}$. In addition, for the combination "JCDU-INTER_CUBIC", the time overhead of our quality enhancement method is only 0.0049 seconds, and for the other combinations, the time overhead of our method is also very small.

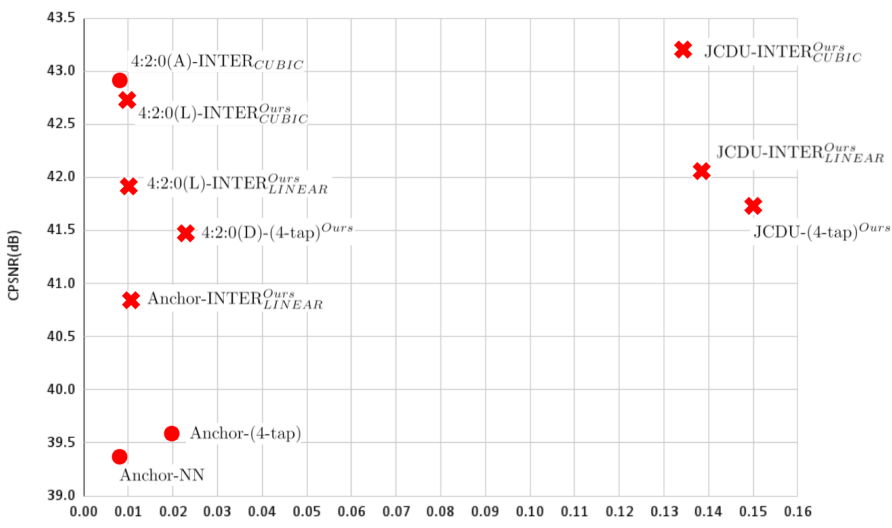

Timess)

Fig. 7. The CPSNR-time diagram of some competitive combinations in $C_{S} \times$ $C_{u}$.

In Fig. 7, the CPSNR-time diagram is provided for ten competitive combinations in $C_{s} \times C_{u}$. The three combinations, 4:2:0(A)-INTER_CUBIC, Anchor-(4-tap), and Anchor-NN, which are marked by red bullets, are CD-free. The seven combinations, 4:2:0(L)-INTER_CUBIC ${ }^{\text {Ours }}$, 4:2:0(L)-INTER_LINEAR ${ }^{\text {Ours }}, \quad$ 4:2:0(D)-(4-tap) ${ }^{\text {Ours }}$, Anchor-INTER_LINEAR ${ }^{\text {Ours }}$, JCDU-INTER_CUBIC ${ }^{\text {Ours }}$,
JCDU-INTER_LINEAR ${ }^{\text {Ours }}$, and JCDU-(4-tap) ${ }^{\text {Ours }}$, which are marked by red crosses, are also CD-free by using our quality enhancement method. From Fig. 7, we observe that if the users prefer the CPSNR performance, they could select the combination JCDU-INTER_CUBIC ${ }^{\text {Ours }}$. On the contrary, if the users prefer the time performance, they could select the VVC-supported combination Anchor-NN. In summary, the CPSNR-time diagram can provide users with more combination options.

\section{B. Quality-bitrate Tradeoff and Visual Effect Merits}

When setting $\mathrm{QP}=0,4,8,12,16,20,24,28,32,36,40$, 44 , and 48 , in terms of the RD-curves (rate-distortion curves), we first demonstrate the quality-bitrate tradeoff merit of our quality enhancement method. Next, the BD-rate (Bjøntegaard delta rate difference) and BD-CPSNR metrics are used to demonstrate the quantity-bitrate merit of our quality enhancement method. Finally, the visual effect merit of our quality enhancement method is demonstrated.

TABLE V

THE QUALITY GAIN AND TIME OVERHEAD OF OUR QUALITY ENHANCEMENT METHOD FOR THE 17 COMBINATIONS IN $S_{C D}$.

\begin{tabular}{|c|c|c|c|c|c|c|c|}
\hline \multicolumn{2}{|c|}{ Combination } & CPSNR & CPSNR gain & SSIMc & SSIMc gain & Time & Time overhead \\
\hline 4:2:0(A) & 4-tap & $\begin{array}{c}40.5558 \\
(41.2976)\end{array}$ & 0.7418 & $\begin{array}{c}0.9800 \\
(0.9819) \\
\end{array}$ & 0.0019 & $\begin{array}{c}0.0184 \\
(0.0208)\end{array}$ & 0.0024 \\
\hline \multirow{2}{*}{$4: 2: 0(\mathrm{~L})$} & INTER_LINEAR & $\begin{array}{c}41.0359 \\
(41.9262)\end{array}$ & 0.8903 & $\begin{array}{c}0.9810 \\
(0.9825)\end{array}$ & 0.0015 & $\begin{array}{c}0.0066 \\
(0.0088)\end{array}$ & 0.0022 \\
\hline & INTER_CUBIC & $\begin{array}{c}41.1022 \\
(42.7300)\end{array}$ & 1.6278 & $\begin{array}{c}0.9813 \\
(0.9842) \\
\end{array}$ & 0.0029 & $\begin{array}{c}0.0065 \\
(0.0091)\end{array}$ & 0.0026 \\
\hline \multirow{3}{*}{$4: 2: 0(\mathrm{R})$} & INTER_LINEAR & $\begin{array}{c}40.8959 \\
(41.5976)\end{array}$ & 0.7017 & $\begin{array}{c}0.9807 \\
(0.9822)\end{array}$ & 0.0015 & $\begin{array}{c}0.0058 \\
(0.0085)\end{array}$ & 0.0027 \\
\hline & INTER_CUBIC & $\begin{array}{c}41.1991 \\
(42.4706)\end{array}$ & 1.2715 & $\begin{array}{c}0.9811 \\
(0.9840)\end{array}$ & 0.0029 & $\begin{array}{c}0.0058 \\
(0.0082)\end{array}$ & 0.0024 \\
\hline & 4-tap & $\begin{array}{c}38.2918 \\
(41.1898)\end{array}$ & 2.8980 & $\begin{array}{c}0.9725 \\
(0.9821) \\
\end{array}$ & 0.0096 & $\begin{array}{c}0.0174 \\
(0.0198)\end{array}$ & 0.0024 \\
\hline \multirow{3}{*}{ 4:2:0(D) } & INTER_LINEAR & $\begin{array}{c}40.1357 \\
(41.9048)\end{array}$ & 1.7691 & $\begin{array}{c}0.9790 \\
(0.9823)\end{array}$ & 0.0033 & $\begin{array}{c}0.0057 \\
(0.0085)\end{array}$ & 0.0028 \\
\hline & INTER_CUBIC & $\begin{array}{c}39.7003 \\
(42.4640)\end{array}$ & 2.7637 & $\begin{array}{c}0.9772 \\
(0.9837)\end{array}$ & 0.0065 & $\begin{array}{c}0.0060 \\
(0.0113)\end{array}$ & 0.0053 \\
\hline & 4-tap & $\begin{array}{c}40.1388 \\
(41.4597)\end{array}$ & 1.3209 & $\begin{array}{c}0.9789 \\
(0.9818) \\
\end{array}$ & 0.0029 & $\begin{array}{c}0.0175 \\
(0.0201) \\
\end{array}$ & 0.0026 \\
\hline \multirow{3}{*}{ IDID [19] } & INTER_LINEAR & $\begin{array}{c}40.4772 \\
(41.2970)\end{array}$ & 198 & $\begin{array}{c}0.9797 \\
(0.9834)\end{array}$ & 0037 & $\begin{array}{c}9.8118 \\
(9.8142)\end{array}$ & 0.0024 \\
\hline & INTER_CUBIC & $\begin{array}{c}39.7991 \\
(42.8056)\end{array}$ & 3.0065 & $\begin{array}{c}0.9769 \\
(0.9841)\end{array}$ & 0.0072 & $\begin{array}{c}9.7466 \\
(9.7491)\end{array}$ & 0.0025 \\
\hline & 4-tap & $\begin{array}{c}40.2789 \\
(41.6577)\end{array}$ & 1.3788 & $\begin{array}{c}0.9788 \\
(0.9820)\end{array}$ & 0.0032 & $\begin{array}{c}9.8881 \\
(9.8908)\end{array}$ & 0.0027 \\
\hline \multirow{3}{*}{ JCDU [17] } & INTER_LINEAR & $\begin{array}{c}40.5021 \\
(42.0679)\end{array}$ & 1.5658 & $\begin{array}{c}0.9797 \\
(0.9826)\end{array}$ & 0.0029 & $\begin{array}{c}0.1287 \\
(0.1320)\end{array}$ & 0.0033 \\
\hline & INTER_CUBIC & $\begin{array}{c}40.4370 \\
(43.2027)\end{array}$ & 2.7657 & $\begin{array}{c}0.9790 \\
(0.9848)\end{array}$ & 0.0058 & $\begin{array}{c}0.1293 \\
(0.1318)\end{array}$ & 0.0025 \\
\hline & 4-tap & $\begin{array}{c}40.5674 \\
(41.7264)\end{array}$ & 1.1590 & $\begin{array}{c}0.9799 \\
(0.9826) \\
\end{array}$ & 0.0027 & $\begin{array}{c}0.1402 \\
(0.1429) \\
\end{array}$ & 0.0027 \\
\hline \multirow{2}{*}{ Anchor } & INTER_LINEAR & $\begin{array}{c}40.2714 \\
(40.8436)\end{array}$ & 0.5722 & $\begin{array}{c}0.9808 \\
(0.9820)\end{array}$ & 0.0012 & $\begin{array}{l}0.0068 \\
(0.095)\end{array}$ & 0.0027 \\
\hline & INTER_CUBIC & $\begin{array}{c}40.6685 \\
(41.7052) \\
\end{array}$ & 1.0367 & $\begin{array}{c}0.9819 \\
(0.9842) \\
\end{array}$ & 0.0023 & $\begin{array}{l}0.0068 \\
(0.094)\end{array}$ & 0.0026 \\
\hline
\end{tabular}

1) The quality-bitrate tradeoff merit in terms of $R D$ curves: Without the loss of generality, we only take the four representative combinations, namely IDID-INTER_CUBIC, JCDU-INTER_CUBIC, 4:2:0(R)-(4-tap), and AnchorINTER_CUBIC, to show the quality-bitrate tradeoff merit of our quality enhancement method. The four blue RD curves in Fig. 8 illustrate the quality-bitrate tradeoff performance of the above four combinations, where the $\mathrm{X}$-axis denotes the average bitrate required for one image and the Y-axis denotes the average CPSNR of the reconstructed RGB full-color images.

After deploying our CDC-based quality enhancement method in the above four combinations, the four enhanced combinations are denoted by IDID-INTER_CUBIC ${ }^{\text {Ours }}$, JCDU-INTER_CUBIC ${ }^{\text {Ours }}, \quad$ 4:2:0(R)-(4-tap) ${ }^{\text {Ours }}, \quad$ and 


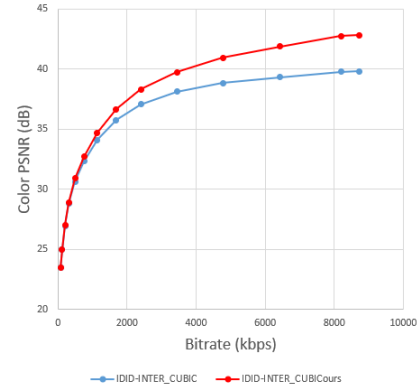

(a)

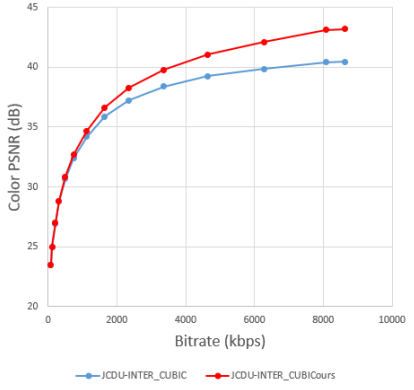

(b)

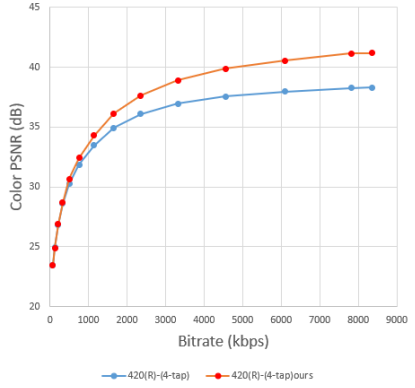

(c)

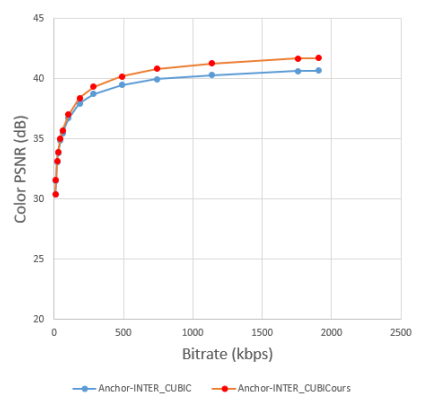

(d)

Fig. 8. In terms of RD curves, the quality-bitrate tradeoff merit of our CDC-based quality enhancement method. (a) For IDID-INTER_CUBIC and IDIDINTER_CUBIC ${ }^{\text {Ours }}$. (b) For JCDU-INTER_CUBIC and JCDU-INTER_CUBIC Ours . (c) For 4:2:0(R)-(4-tap) and 4:2:0(R)-(4-tap) ${ }^{\text {Ours }}$. (d) For AnchorINTER_CUBIC and Anchor-INTER_CUBIC ${ }^{\bar{O} u r s}$.

Anchor-INTER_CUBIC ${ }^{\text {Ours }}$. The four RD curves of the four enhanced combinations are depicted by the red curves in Fig. 8. From Fig. 8, we observe that under the same bitrate condition, each enhanced combination always has much better quality relative to the original combination, indicating the quality-bitrate tradeoff merit of our quality enhancement method.

TABLE VI

THE BD-RATE REDUCTION (\%) MERIT OF OUR QUALITY ENHANCEMENT METHOD FOR THE 17 COMBINATIONS IN $S_{C D}$

\begin{tabular}{|c|c|c|c|c|c|c|}
\hline \multicolumn{2}{|c|}{$I^{R G B}$} & QP 4-16 & QP $12-24$ & QP 20-32 & QP $28-40$ & QP $36-48$ \\
\hline $4: 2: 0(\mathrm{~A})$ & 4-tap & -20.6402 & -10.2438 & -4.0850 & -1.2376 & 0.3934 \\
\hline \multirow{2}{*}{$4: 2: 0(\mathrm{~L})$} & INTER_LINEAR & -19.0471 & -10.0621 & -4.9057 & -2.6350 & -1.1220 \\
\hline & INTER_CUBIC & -37.1418 & -26.6727 & -19.2333 & -17.2882 & -18.3661 \\
\hline \multirow{3}{*}{$4: 2: 0(\mathrm{R})$} & INTER_LINEAR & -16.7687 & -8.7327 & -3.9057 & -1.5365 & 0.2820 \\
\hline & INTER_CUBIC & -26.0285 & -13.7237 & -6.2897 & -2.6358 & 0.1537 \\
\hline & 4-tap & -69.5603 & -39.5169 & -19.8765 & -10.0628 & -1.9208 \\
\hline \multirow{3}{*}{$4: 2: 0(D)$} & INTER_LINEAR & -38.2216 & -19.5560 & -8.9567 & -4.2877 & -1.3053 \\
\hline & INTER_CUBIC & -54.4535 & -28.5779 & -13.5396 & -6.7323 & -2.0848 \\
\hline & 4-tap & -33.7705 & -15.6471 & -6.3031 & -2.4761 & -0.3009 \\
\hline \multirow{3}{*}{ IDID [19] } & INTER_LINEAR & -40.0060 & -20.8197 & -9.5580 & -4.4025 & -1.2278 \\
\hline & INTER_CUBIC & -57.4937 & -30.8381 & -14.7352 & -7.0842 & -2.0610 \\
\hline & 4-tap & -35.8717 & -16.8786 & -6.8286 & -2.4960 & -0.1655 \\
\hline \multirow{3}{*}{ JCDU [17] } & INTER_LINEAR & -32.8419 & -17.0514 & -7.6134 & -3.3463 & -0.8827 \\
\hline & INTER_CUBIC & -48.1825 & -25.9264 & -12.0611 & -5.4898 & -1.4992 \\
\hline & 4-tap & -28.8022 & -13.7425 & -5.3237 & -1.7216 & 0.0361 \\
\hline \multirow{2}{*}{ Anchor } & INTER_LINEAR & -43.0566 & -22.7710 & -10.4923 & -5.6666 & -2.4207 \\
\hline & INTER_CUBIC & -57.6304 & -31.4548 & -14.6227 & -7.7802 & -3.1640 \\
\hline
\end{tabular}

2) The BD-rate and BD-CPSNR merits: BD-rate indicates the average bitrate reduction percentage under the same quality circumstance, and BD-CPSNR indicates the average CPSNR gain percentage under the same bitrate circumstance. When setting four QP intervals, namely 4-16, 12-24, 20-32, 28-40, and 36-48, the BD-rate and BD-CPSNR metrics are used to demonstrate the quality-bitrate tradeoff merits of our quality enhancement method.

Based on the four datasets, Table VI indicates that the average $\mathrm{BD}$-rate reduction percentage of the reconstructed RGB full-color images using our quality enhancement method for the concerned 17 combinations is $-15.3247 \%$. In Table VII, the average BD-CPSNR gain $(\mathrm{dB})$ of the reconstructed RGB full-color images using our quality enhancement method for the same 17 combinations is $0.5661 \mathrm{~dB}$.

TABLE VII

THE BD-CPSNR GAIN (dB) MERIT OF OUR QUALITY ENHANCEMENT METHOD FOR THE 17 COMBINATIONS IN $S_{C D}$.

\begin{tabular}{|c|c|c|c|c|c|c|}
\hline \multicolumn{2}{|c|}{$I^{R G B}$} & QP 4-16 & QP $12-24$ & QP 20-32 & QP $28-40$ & QP $36-48$ \\
\hline $4: 2: 0(\mathrm{~A})$ & 4-tap & 0.5993 & 0.3926 & 0.1915 & 0.0560 & -0.0158 \\
\hline \multirow{2}{*}{$4: 2: 0(\mathrm{~L})$} & INTER_LINEAR & 0.6361 & 0.4094 & 0.2338 & 0.1188 & 0.0470 \\
\hline & INTER_CUBIC & 1.2722 & 1.1514 & 0.9949 & 0.8402 & 0.8305 \\
\hline \multirow{3}{*}{ 4:2:0(R) } & INTER_LINEAR & 0.5388 & 0.3491 & 0.1839 & 0.0685 & -0.0108 \\
\hline & INTER_CUBIC & 0.9558 & 0.6074 & 0.3137 & 0.1204 & -0.0051 \\
\hline & 4-tap & 2.4034 & 1.7265 & 1.0048 & 0.4603 & 0.0857 \\
\hline \multirow{3}{*}{ 4:2:0(D) } & INTER_LINEAR & 1.3367 & 0.8349 & 0.4362 & 0.193 & 0.0546 \\
\hline & INTER_CUBIC & 2.0939 & 1.3274 & 0.6962 & 0.3101 & 0.0885 \\
\hline & 4-tap & 1.0139 & 0.6322 & 0.3035 & 0.1115 & 0.0129 \\
\hline \multirow{3}{*}{ IDID [19] } & INTER_LINEAR & 1.4836 & 0.9148 & 0.4719 & 0.2021 & 0.0524 \\
\hline & INTER_CUBIC & 2.2751 & 1.4462 & 0.7611 & 0.3317 & 0.0894 \\
\hline & 4-tap & 1.0706 & 0.6786 & 0.3284 & 0.1140 & 0.0075 \\
\hline \multirow{3}{*}{ JCDU [17] } & INTER_LINEAR & 1.1612 & 0.7140 & 0.3658 & 0.1519 & 0.0374 \\
\hline & INTER_CUBIC & 2.0005 & 1.2212 & 0.6235 & 0.2582 & 0.0650 \\
\hline & 4-tap & 0.8874 & 0.5484 & 0.2551 & 0.0789 & -0.0011 \\
\hline \multirow{2}{*}{ Anchor } & INTER_LINEAR & 0.5089 & 0.3835 & 0.2386 & 0.1353 & 0.0654 \\
\hline & INTER_CUBIC & 0.8914 & 0.6397 & 0.3734 & 0.1971 & 0.0882 \\
\hline
\end{tabular}

3) The visual effect merit: We take the 7th ground truth Kodak image in Fig. 9(a) as the testing image to demonstrate the visual effect merit of the reconstructed RGB full-color image by deploying our quality enhancement method in the above four comparative combinations. The magnified subimage in Fig. 9(b) is decoupled from the color region containing the fence in Fig. 9(a).

For $\mathrm{QP}=48$, after performing the eight considered combinations on Fig. 9(a), the eight reconstructed magnified subimages for Fig. 9(b) are shown in Figs. 9(c)-(j). As depicted in 


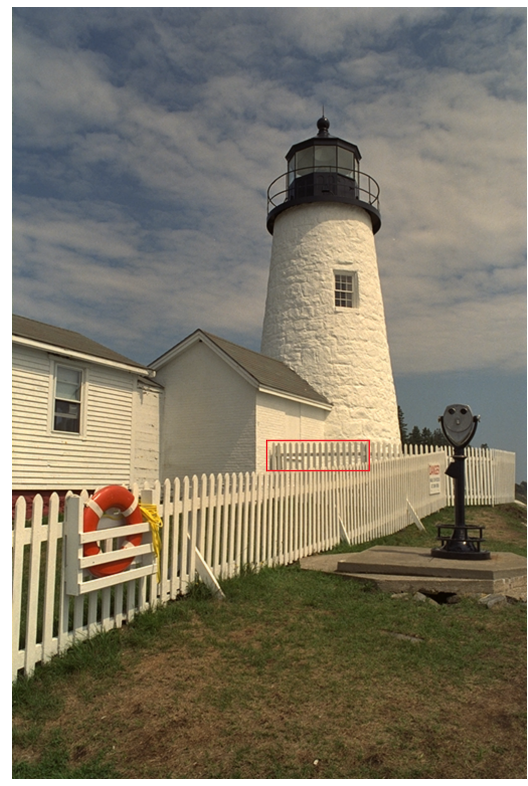

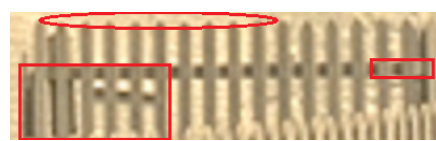

(b)

(a)

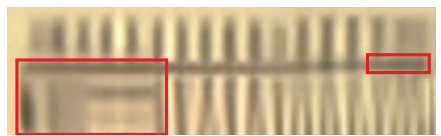

(c)

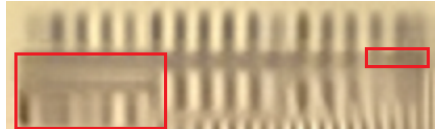

(g)

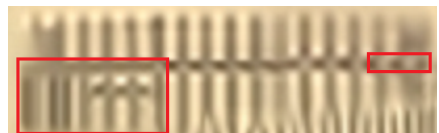

(d)

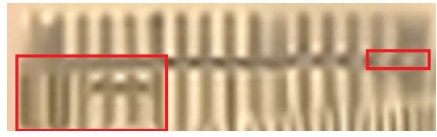

(h)

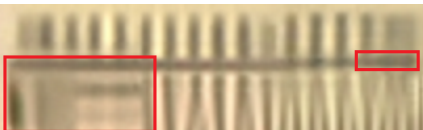

(e)

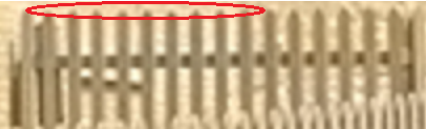

(i)

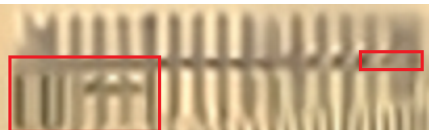

(f)

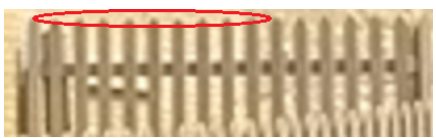

(j)

Fig. 9. The visual effect merit of our quality enhancement method. (a) The 7th ground truth Kodak image. (b) The magnified subimage of (a). (c) IDIDINTER_CUBIC. (d) IDID-INTER_CUBICOurs. (e) JCDU-INTER_CUBIC. (f) JCDU-INTER_CUBICOurs . (g) 4:2:0(R)-(4-tap). (h) 4:2:0(R)-(4-tap) Ours . (i) Anchor-INTER_CUBIC. (j) Anchor-INTER_CUBICOurs .

the red rectangles of Fig. 9(d), Fig. 9(f), and Fig. 9(h), the three improved combinations, IDID-INTER_CUBIC ${ }^{\text {Ours }}$, JCDU-INTER_CUBIC ${ }^{\text {Ours }}$, and 4:2:0(R)-(4-tap) ${ }^{\text {Ours }}$, have clear visual and texture-preserving improvements relative to Fig. 9(c), Fig. 9(e), and Fig. 9(g), respectively. As depicted in the red ellipse of Fig. 9(j), our quality enhancement method has a better texture-preserving effect relative to Fig. 9(i) using the original combination "Anchor-INTER_CUBIC", while for the other parts of the two reconstructed RGB full-color images, both methods are very close to each other.

\section{CONCLUSION}

We have presented the proposed CDC-based qualityenhancement method in the augmented coding system to eliminate the $\mathrm{CD}$ bias problem happening in combinations in the current coding system. First, we define the subsampled chroma positions of the considered seven chroma subsampling methods. Next, we analyze the $\mathrm{CD}$ bias problem occurring between the subsampled $\mathrm{CbCr}$ image $I^{s u b}, \mathrm{CbCr}$ which is prepared for compression at the server side. For each combination in $C_{s} \times C_{u}$, from the server side to the client side, the overall CDV of that combination is analyzed. In particular, we explain why the $\mathrm{CD}$ bias problem between the upsampled $\mathrm{CbCr}$ image
$I^{u p, C b C r}$ and the luma image $I^{Y}$ for any combination in $\mathrm{S}_{C D}$ leads to the quality degradation of the reconstructed RGB full-color image. Further, we propose a CDC-based quality enhancement method to solve the $\mathrm{CD}$ bias problem for each combination in $\mathrm{S}_{C D}$ to enhance the quality of the reconstructed RGB full-color image.

Based on four datasets, the comprehensive experimental results have demonstrated that our quality enhancement method achieves significant quality and the quality-bitrate tradeoff improvements on the newly released VVC platform VTM12.0. The future work is to apply the result of this paper to tackle the following issues: the luma-guided chroma component coding [6], the luma-guided chroma reconstruction [14], the DCT-based chroma subsampling and upsampling [20], and the cross-space distortion directed image coding [21], for improving the quality of the reconstructed images.

\section{ACKNOWLEDGMENT}

The authors appreciate the proofreading help of Ms. C. Harrington to improve the manuscript.

\section{APPENDIX A}

In this Appendix, during the description of the 4-tap filter, which consists of two steps, to reconstruct each $2 \times 2 \mathrm{CbCr}$ 


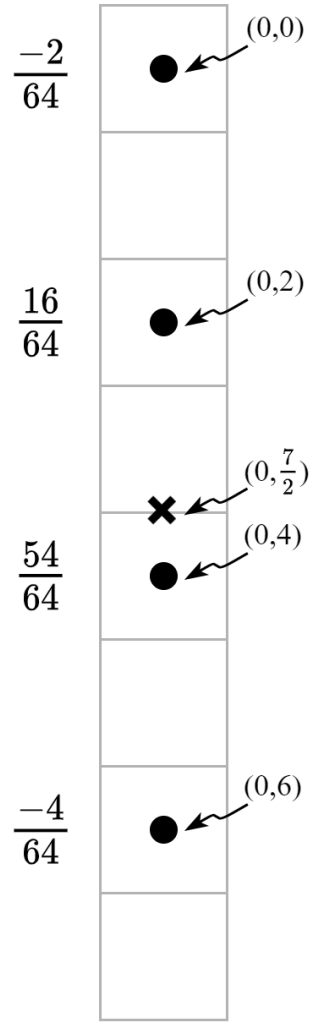

(a)

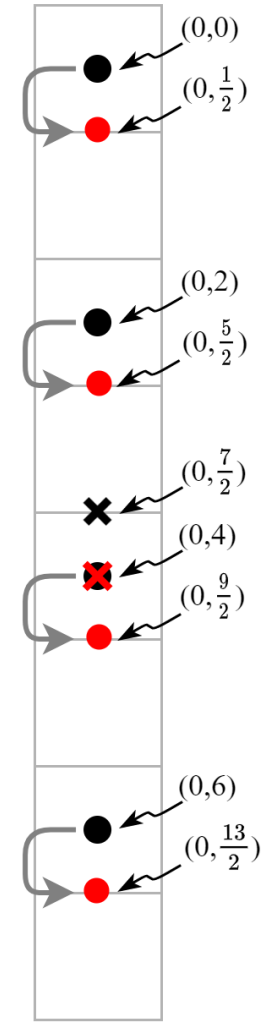

(b)
Fig. 10. The balance point of 4-tap and how the 4-tap method eliminates the nonzero CDV occurring in Anchor at the server side. (a) The balance point of the 4-tap filter is located at $\left(0, \frac{7}{2}\right)$. (b) Eliminating the nonzero CDV occurring in Anchor at the server side by moving half a pixel downward.

block, we utilize the moment balance law to explain why the nonzero CDV $\left(=\left(0,-\frac{1}{2}\right)\right)$ occurring in the chroma subsampling method "Anchor" used at the server side can be eliminated by the 4-tap filter such that the resultant CDV of the combination "Anchor-(4-tap)" equals $(0,0)$. On the other hand, we utilize the moment balance law to explain why the VVC-supported combination "Anchor-(4-tap)" is CD-free.

In the first step of the 4-tap method, suppose the 4-tap filter $([-2,16,54,-4] / 64)^{t}$ is performed on the four subsampled chroma pairs, as marked by the four black bullets in Fig. 10(a). Then, as the reconstructed chroma pair, the response is placed at location $(0,4)$. In Fig. 10(a), suppose the four subsampled chroma pairs are located at $(0,0),(0,2),(0,4)$, and $(0,6)$ corresponding to the four coefficients, $-2 / 64,16 / 64,54 / 64$, and $-4 / 64$, respectively. According to the moment balance law [8], the location of the response $\left(=-2 / 64 * I^{\text {initial }, C b C r}(0,0)+\right.$ $16 / 64 * I^{\text {initial }, C b C r}(0,2)+54 / 64 * I^{\text {initial }, C b C r}(0,4)-4 / 64$ * I initial, $\mathrm{CbCr}(0,6))$ should be located at the balance point $(0, y)$. Therefore, it yields

$$
\frac{54}{64} *(4-y)-\frac{4}{64} *(6-y)=\frac{16}{64} *(y-2)-\frac{2}{64} * y
$$

After solving Eq. (8), it yields $y=\frac{7}{2}$. That is, the location of the balance point equals $\left(0, \frac{7}{2}\right)$, which is denoted by a black cross in Fig. 10(a). Relative to the position of the reconstructed chroma pair, namely $(0,4)$, the first step of the 4-tap method yields a $\mathrm{CD}$ bias problem with $\mathrm{CDV}=\left(0, \frac{1}{2}\right)(=(0,4)$ $\left.\left(0, \frac{7}{2}\right)\right)$ (see Table II), just eliminating the CDV $\left(=\left(0,-\frac{1}{2}\right)\right.$ occurring in the chroma subsampling method "Anchor" at the server side, as depicted in Table I. In practice, as depicted in Fig. 10(b), the chroma upsampling method "4-tap" first moves the subsampled chroma pairs $I^{\text {initial }, C b C r}(2 x, 2 y)$ in black to $I^{\text {initial, } C b C r}\left(2 x, 2 y+\frac{1}{2}\right)$ in red, and then it can explain why the response of the first step of the 4-tap method can eliminate the $\mathrm{CDV}=\left(0,-\frac{1}{2}\right)$ occurring in the Anchor method.

By the same argument, the 4-tap method further reconstructs the missing chroma pair located at $(0,5)$ in Fig. 10(a), and the response equals $(=-4 / 64 * \mathrm{I}$ initial, $\mathrm{CbCr}(0$, $2)+54 / 64 * I^{\text {initial }, C b C r}(0,4)+16 / 64 * \mathrm{I}^{\text {initial }, C b C r}(0,6)-$ $\left.2 / 64 * I^{\text {initial }, C b C r}(0,8)\right)$. According to the moment balance law, we can show that the location of the balance point of the response equals $\left(0, \frac{9}{2}\right)$. In order to eliminate the CDV $(=(0,-$ $\frac{1}{2}$ ) occurring in the chroma subsampling method "Anchor" at the server side, the 4-tap method first moves each subsampled chroma pair half a pixel downward, as depicted in Fig. 10(b), and then the related inner product is performed.

In the second step, the 4-tap method reconstructs the missing chroma pair located at $(1,4)$ by using the response: $-4 / 64 * \mathrm{I}^{\text {initial }, \mathrm{CbCr}}(-2,4)+36 / 64 * \mathrm{I}^{\text {initial }, C b C r}(0,4)$ $+36 / 64 * I^{\text {initial, } C b C r}(2,4)-4 / 64 * I^{\text {initial }, C b C r}(4,4)$, where the chroma values of $\mathrm{I}^{\text {initial, } \mathrm{CbCr}}(-2,4)$, Initial, $\mathrm{CbCr}(0,4)$, $\mathrm{I}^{\text {initial, } \mathrm{CbCr}}(2,4)$, and $\mathrm{I}^{\text {initial, } C b C r}(4,4)$ have been updated by the first step. Because the filter $[-4,36,36,-4] / 64$ is symmetric, the balance point of the response is exactly located at (1, 4), causing no CD bias problem. Similarly, the 4-tap method reconstructs the missing chroma pair located at $(1,5)$ by using the response: $-4 / 64 * I^{\text {initial }, C b C r}(-$ $2,5)+36 / 64 * I^{\text {initial }, C b C r}(0,5)+36 / 64 * I^{\text {initial }, C b C r}(2,5)$ - 4/64*Initial,CbCr $(4,5)$.

We have explained how the 4-tap chroma upsampling method works, and have utilized the moment balance law to explain why the VVC-supported combination "Anchor-(4tap)" is CD-free.

\section{REFERENCES}

[1] G. Bjøntegaard, "Calculation of average PSNR difference between RDcurves," ITU-T SG16/Q6 VCEG, Austin, TX, USA, document VECGM33, pp. 2-4, Apr. 2001.

[2] HM-16.18. Accessed: Jan. 2018. [Online]. Available: https://hevc.hhi. fraunhofer.de/svn/svn_HEVCSoftware/tags/HM-16.18/.

[3] "ITU-R Recommendation BT-601-5: Studio encoding parameters of digital television for standard 4:3 and wide-screen 16:9 aspect ratios." International Telecommunications Union, 2011.

[4] IMAX database. http://www4.comp.polyu.edu.hk/ cslzhang/CDM_ Dataset.htm.

[5] Kodak database. http://www.math.purdue.edu/ lucier/PHOTO_CD/BMP_ IMAGES/.

[6] J. Li, M. Wang, L. Zhang, K. Zhang, S. Wang, S. Wang, S. Ma, and W. Gao, "Sub-sampled cross-component prediction for chroma component coding," in Data Compression Conference (DCC), pp. 203-212, Mar. 2020.

[7] X. Li and M. T. Orchard, "New edge-directed interpolation," IEEE Transactions on Image Processing, vol. 10, no. 10, pp. 1521-1527, Oct. 2001.

[8] S. J. Ling, J. Sanny, W. Moebs, University Physics Volume 1, https://assets.openstax.org/oscms-prodcms/media/documents/ UniversityPhysicsVolume1-OP.pdf. 
[9] A. Luthra, E. François, and W. Husak, Call for evidence (CfE) for HDR and WCG video coding, Standard ISO/IEC JTC1/SC29/WG11 (MPEG), Geneva, Switzerland, Feb. 2015.

[10] Open CV book. https://books.google.com.tw/books?hl=zhTW\&lr=\&id=seAgiOfu2EIC\&oi=fnd\&pg=PR3\&dq=opencv+library\& ots=hUL28niGSa\&sig=2f-MT4LGYlFPnyfkVd6xLIo27VM\&redir_esc= $\mathrm{y} \# \mathrm{v}=$ snippet $\& \mathrm{q}=$ bilinear $\& \mathrm{f}=$ false.

[11] Open CV codes. https://sourceforge.net/projects/opencvlibrary/?fbclid= IwAR2vXid_ZecTmGKWiCuOe2XuaCKdAq4-Aes7FfeEVIpDMBwOJAgg6TbI-k.

[12] W. Pu, M. Karczewicz, R. Joshi, V. Seregin, F. Zou, J. Sole, Y. C. Sun, T. D. Chuang, P. Lai, S. Liu, S. T. Hsiang, J. Ye, and Y. W. Huang, "Palette mode coding in HEVC screen content coding extension," IEEE J. Emerging and Selected Topics in Circuits and Systems, vol. 6, no. 4, pp. 420-432, Dec. 2016.

[13] SCI Image Database, accessed on Jan. 10, 2016. [Online]. Available: ftp://140.118.175.164/SCI.

[14] T. Vermeir et al., "Guided chroma reconstruction for screen content coding," IEEE Trans. Circuits Syst. Video Technol., vol. 26, no. 10, pp. 1884-1892, Oct. 2016.

[15] Versatile Video Coding (VVC). Available: https://vcgit.hhi.fraunhofer.de/ jvet/VVCSoftware_VTM.

[16] The Video Dataset. ftp://140.118.175.164/CFASS/

[17] S. Wang, K. Gu, S. Ma, and W. Gao, "Joint chroma downsampling and upsampling for screen content image," IEEE Transactions on Circuits and Systems for Video Technology, vol. 26, no. 9, pp. 1595-1609, Sep. 2016.

[18] Z. Wang, A. C. Bovik, H. R. Sheikh, and E. P. Simoncelli, "Image quality assessment: from error measurement to structural similarity," IEEE Transactions on Image Processing, vol. 13, no. 4, pp. 600-612, Apr. 2004.

[19] Y. Zhang, D. Zhao, J. Zhang, R. Xiong, and W. Gao, "Interpolationdependent image downsampling," IEEE Transactions on Image Processing, vol. 20, no. 11, pp. 3291-3296, Nov. 2011.

[20] S. Zhu, C. Cui, R. Xiong, Y. Guo, and B. Zeng, "Efficient chroma subsampling and luma modification for color image compression," IEEE Transactions on Circuits and Systems for Video Technology, vol. 29, no. 5, pp. 1559-1563, May 2019.

[21] S. Zhu, M. Li, C. Chen, S. Liu, and B. Zeng, "Cross-space distortion directed color image compression," IEEE Transactions on Multimedia, vol. 20, no. 3, pp. 525-538, 2018, Mar. 2018.

[22] Y. Lu, S. Li, and H. Shen, "Virtualized screen: a third element for cloudmobile convergence," IEEE Multimedia Magazine, vol. 18, no. 2, pp. 4-11, Feb. 2011.

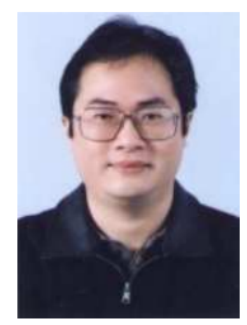

Kuo-Liang Chung (SM01) received his B.S., M.S., and Ph.D. degrees from National Taiwan University, Taipei, Taiwan in 1982, 1984, and 1990, respectively. $\mathrm{He}$ has been one Chair Professor of the Department of Computer Science and Information Engineering at National Taiwan University of Science and Technology, Taipei, Taiwan since 2009. He was the recipient of the Distinguished Research Award (2004-2007; 2019-2022) and Distinguished Research Project Award (2009-2012) from the Ministry of Science and Technology of Taiwan. He has been an Associate Editor of the Journal of Visual Communication and Image Representation since 2011. His research interests include machine learning, image processing, and video compression.

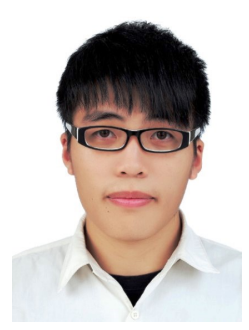

Chen-Wei Kao received his B.S. degree in Computer Science and Engineering from the National Taiwan Ocean University, Keelung, Taiwan, in 2019. $\mathrm{He}$ is currently working towards his M.S. degree in Computer Science and Information Engineering at the National Taiwan University of Science and Technology, Taipei, Taiwan. His research interests include image processing and video compression. 